\title{
Hesitant Pythagorean fuzzy interaction aggregation operators and their application in multiple attribute decision-making
}

\author{
Wei Yang ${ }^{1}\left(\mathbb{D} \cdot\right.$ Chengjun Wang $^{1} \cdot{\text { Yong } \text { Liu }^{1} \cdot \text { Yan Sun }}^{1}$
}

Received: 12 September 2018 / Accepted: 5 April 2019 / Published online: 8 May 2019

(c) The Author(s) 2019

\begin{abstract}
The aim of this paper is to develop hesitant Pythagorean fuzzy interaction aggregation operators based on the hesitant fuzzy set, Pythagorean fuzzy set and interaction between membership and non-membership. The new operation laws can overcome shortcomings of existing operation laws of hesitant Pythagorean fuzzy values. Several new hesitant Pythagorean fuzzy interaction aggregation operators have been developed including the hesitant Pythagorean fuzzy interaction weighted averaging operator, the hesitant Pythagorean fuzzy interaction weighted geometric averaging operator and the generalized hesitant Pythagorean fuzzy interaction weighted averaging operator. Using the Bonferroni mean, some hesitant Pythagorean fuzzy interaction Bonferroni mean operators have been developed including the hesitant Pythagorean fuzzy interaction Bonferroni mean operator, the hesitant Pythagorean fuzzy interaction weighted Bonferroni mean (HPFIWBM) operator, the hesitant Pythagorean fuzzy interaction geometric Bonferroni mean operator and the hesitant Pythagorean fuzzy interaction geometric weight Bonferroni mean (HPFIGWBM) operator. Some properties have been studied. A new multiple attribute decision-making method based on the HPFIWBM operator and the HPFIGWBM operator has been presented. Numerical example is presented to illustrate the new method.
\end{abstract}

Keywords Hesitant fuzzy set $\cdot$ Pythagorean fuzzy set $\cdot$ Multiple attribute decision-making $\cdot$ Bonferroni mean

\section{Introduction}

Fuzzy decision-making has been studied and applied extensively [1-3]. Pythagorean fuzzy set [4,5] is the extension of intuitionistic fuzzy set [6]. In intuitionistic fuzzy set, the sum of membership and non-membership is no more than 1, while in Pythagorean fuzzy set, the square sum of membership and non-membership is no more than 1 . Hence, Pythagorean fuzzy set has larger feasible region than that of intuitionistic fuzzy set. Thus, it is more powerful and flexible in modeling fuzzy and uncertain information. Pythagorean fuzzy set has been studied and applied extensively [7-21]. Some aggregation operators have been developed in Pythagorean fuzzy environment. Liang et al. [22] proposed the Pythagorean fuzzy Bonferroni mean operator and the weighted Pythagorean fuzzy Bonferroni mean operator. Zhang et al. [23] presented some general-

Wei Yang

yangweipyf@163.com; yangwei_lxy@xauat.edu.cn

1 Xi' an University of Architecture and Technology, Xi' an 710055, Shaanxi, People's Republic of China ized Pythagorean fuzzy Bonferroni mean operator and the generalized Pythagorean fuzzy Bonferroni geometric mean operator. Yang and Pang [24] developed some Pythagorean fuzzy interaction Maclaurin symmetric mean operators. Rahman et al. [25] defined some interval-valued Pythagorean fuzzy aggregation operators including the interval-valued Pythagorean fuzzy weighted geometric operator, the intervalvalued Pythagorean fuzzy ordered weighted geometric operator, and the interval-valued Pythagorean fuzzy hybrid geometric operator. Wei and Lu defined some Pythagorean fuzzy power aggregation operators in [26] and presented some dual hesitant Pythagorean fuzzy aggregation operators in [27]. Garg presented the generalized Pythagorean fuzzy Einstein weighted average operator and the generalized Pythagorean fuzzy Einstein ordered weighted average operator in [28] and developed the Pythagorean fuzzy geometric interactive aggregation operators using Einstein operations in [29]. Du et al. [30] defined interval-valued Pythagorean fuzzy linguistic variable set and defined interval-valued Pythagorean fuzzy linguistic ordered weighted averaging operator and generalized interval-valued Pythagorean fuzzy linguistic ordered weighted average operator. Wei [31] defined some 
Pythagorean fuzzy interaction aggregation operators and some Pythagorean fuzzy interaction geometric aggregation operators. Some multiple attribute decision-making methods in Pythagorean fuzzy environment have been developed. Zhang and $\mathrm{Xu}$ [32] extended the TOPSIS method to accommodate Pythagorean fuzzy values. Ren et al. [33] proposed Pythagorean fuzzy TODIM approach. Chen [34] presented Pythagorean fuzzy VIKOR methods based on the generalized Pythagorean fuzzy distance measure. Pythagorean fuzzy set has been extended to accommodate interval values $[35,36]$, linguistic arguments [37,38], probabilistic information [39], etc.

Hesitant fuzzy set [40] is the extension of fuzzy set and intuitionistic fuzzy set. In hesitant fuzzy set, each membership may include several possible values. Hesitant fuzzy set has been extended to accommodate intuitionistic fuzzy set [41], linguistic arguments [42], linguistic intuitionistic fuzzy values [43-47]. Hesitant Pythagorean fuzzy sets was defined [48] and some hesitant Pythagorean fuzzy Hamacher aggregation operators have been developed including the hesitant Pythagorean fuzzy Hamacher weighted average operator, hesitant Pythagorean fuzzy Hamacher weighted geometric operator. Khan et al. [49] proposed maximizing deviation method for Pythagorean hesitant fuzzy numbers in which information about attribute weights is incomplete. Garg [50] defined some hesitant Pythagorean fuzzy weighted aggregation operators and hesitant Pythagorean fuzzy geometric aggregation operators. But in real decision-making process, there are still cases that can not be dealt with using existing methods. For example, in evaluating some car, the experts gave evaluation values of power, noise and speed as $\{(0.9,0.0)\},\{(0.6,0.5)\},\{(0.8,0.3),(0.7,0.4)\}$. If the attribute weight vector is $(0.25,0.35,0.40)$. Then, using the existing operation laws of hesitant Pythagorean fuzzy values, the weighted averaging values can be calculated as $\{(0.7904,0),(0.7541,0)\}$. There is only 0 non-membership and the other two non-memberships are not 0 , but they have no effect on the final results. To overcome this shortcoming, we propose some interaction operation laws for hesitant Pythagorean fuzzy values by considering interaction between membership and non-membership. Then, we first develop some aggregation operators including the hesitant Pythagorean fuzzy interaction weighted averaging (HPFIWA) operator, hesitant Pythagorean fuzzy interaction weighted geometric averaging (HPFIWGA) operator and the generalized hesitant Pythagorean fuzzy interaction weighted geometric averaging (GHPFIWA) operator. The Bonferroni mean was first introduced by Bonferroni [51], which can capture inter-relationship among arguments to be aggregated. Yager [52] provided an interpretation of Bonferroni mean as involving a product of each argument with the average of the other arguments. Beliakov et al. [53] developed generalized Bonferroni mean. Beliakov and
James [54] extended the generalized Bonferroni mean to intuitionistic fuzzy environment. $\mathrm{Zhu}$ and $\mathrm{Xu}$ [55] proposed the hesitant fuzzy Bonferroni mean operator. Yang et al. [56] developed the Pythagorean fuzzy interaction partitioned Bonferroni mean operator. But Bonferroni mean for hesitant Pythagorean values considering interaction between membership and non-membership has not been studied yet. Yang et al. [57] proposed q-rung orthopair fuzzy partitioned Bonferroni mean operators. To model interaction among hesitant Pythagorean fuzzy values and interaction between membership and non-membership at the same time, we develop some hesitant Pythagorean fuzzy interaction Bonferroni mean operator including the hesitant Pythagorean fuzzy interaction Bonferroni mean (HPFIBM) operator, the hesitant Pythagorean fuzzy interaction weighted Bonferroni mean (HPFIWBM) operator, the hesitant Pythagorean fuzzy interaction geometric Bonferroni mean (HPFIGBM) operator and the hesitant Pythagorean fuzzy interaction weighted geometric Bonferroni mean (HPFIWGBM) aggregation operator.

The objective of the paper is to develop some hesitant Pythagorean fuzzy interaction Bonferroni mean operators. To do so, the structure of the paper is as follows. In "Preliminaries", some basic concepts on Pythagorean fuzzy set, hesitant fuzzy set have been reviewed. Some interaction operational laws for hesitant Pythagorean fuzzy values have been defined and some properties have been studied. In "Hesitant Pythagorean fuzzy interaction aggregation operators", some hesitant Pythagorean fuzzy interaction aggregation operators have been defined. In "Hesitant Pythagorean fuzzy interaction Bonferroni mean operators", some hesitant Pythagorean fuzzy interaction Bonferroni mean operators have been proposed. In "An approach to Pythagorean fuzzy multiple attribute decision-making based on new interaction aggregation operators", a new multiple attribute decision-making method based on the HPFIWBM operator and the HPFIWGBM operator has been presented. In "An illustrative example", numerical example is presented to illustrate the new method. Conclusions are presented in the final section.

\section{Preliminaries}

Definition 1 [40] Let $X$ be a fixed set. A hesitant fuzzy set (HFS) $H$ on $X$ in terms of a function that when applied to $X$ returns a subset of $[0,1]$,

$H=\left\{\left\langle x, h_{H}(x)\right\rangle \mid x \in X\right\}$,

where $h_{H}(x)$ is a set of values in $[0,1]$, denoting the possible membership degrees of element $x \in X$ to set $H$. For convenience, $h_{H}(x)$ is called a hesitant fuzzy element (HFE).

Definition 2 [4] Let $X$ be a fixed set. A Pythagorean fuzzy set $P$ on $X$ can be represented as follows 
$P=\left\{\left\langle x,\left(\mu_{P}(x), v_{P}(x)\right)\right\rangle \mid x \in X\right\}$,

where $\mu_{P}(x): X \rightarrow[0,1]$ is the membership function and $v_{P}(x): X \rightarrow[0,1]$ is the non-membership function. For each $x \in X$, it satisfies the following condition $0 \leq\left(\mu_{P}(x)\right)^{2}+\left(\nu_{P}(x)\right)^{2} \leq 1 . \pi_{P}(x)=$ $\sqrt{1-\left(\mu_{P}(x)\right)^{2}-\left(v_{P}(x)\right)^{2}}$ is the indeterminacy degree of $x$ to $X$. For simplicity, $\left(\mu_{P}(x), v_{P}(x)\right)$ is called a Pythagorean fuzzy number $(\mathrm{PFN})$, denoted by $\left(\mu_{P}, v_{P}\right)$, where $\mu_{P}, v_{P} \in$ $[0,1], \pi_{P}=\sqrt{1-\left(\mu_{P}\right)^{2}-\left(v_{P}\right)^{2}}$ and $0 \leq\left(\mu_{P}\right)^{2}+\left(v_{P}\right)^{2} \leq$ 1.

Definition 3 [32] Let $\alpha=\left(\mu_{\alpha}, v_{\alpha}\right), \alpha_{1}=\left(\mu_{1}, v_{1}\right)$ and $\alpha_{2}=$ $\left(\mu_{2}, \nu_{2}\right)$ be three PFNs, the operations are as follows

(1) $\alpha_{1} \oplus \alpha_{2}=\left(\sqrt{\mu_{1}^{2}+\mu_{2}^{2}-\mu_{1}^{2} \mu_{2}^{2}}, v_{1} v_{2}\right)$,

(2) $\alpha_{1} \otimes \alpha_{2}=\left(\mu_{1} \mu_{2}, \sqrt{v_{1}^{2}+v_{2}^{2}-v_{1}^{2} v_{2}^{2}}\right)$,

(3) $k \alpha=\left(\sqrt{1-\left(1-\mu^{2}\right)^{k}},(v)^{k}\right), k \geq 0$,

(4) $\alpha^{k}=\left(\mu^{k}, \sqrt{1-\left(1-v^{2}\right)^{k}}\right), k \geq 0$.

Definition 4 [48,50] Let $X$ be a fixed set. A hesitant Pythagorean fuzzy set $\tilde{P}$ on $X$ can be represented as follows

$\tilde{P}=\left\{\left\langle x,\left(\tilde{h}_{P}(x), \tilde{g}_{P}(x)\right)\right\rangle \mid x \in X\right\}$,

where $\tilde{h}_{P}(x)=\left\{\mu_{i}\right\}$ is the set of all the possible memberships of element $x \in X$ and $\tilde{g}_{P}(x)=\left\{v_{i}\right\}$ is the set of all the possible non-memberships of element $x \in X$, $\mu_{i} \in[0,1], v_{i} \in[0,1]$. Let $\mu^{+}=\max \left\{\mu_{i}\right\}, v^{+}=\max \left\{v_{i}\right\}$, $\left(\mu^{+}\right)^{2}+\left(v^{+}\right)^{2} \leq 1$. For convenience, $\left(\tilde{h}_{P}(x), \tilde{g}_{P}(x)\right)$ is called a hesitant Pythagorean fuzzy element (HPFE).

Definition 5 [50] Let $\tilde{f}=(\tilde{h}, \tilde{g}), \tilde{f}_{1}=\left(\tilde{h}_{1}, \tilde{g}_{1}\right), \tilde{f}_{2}=$ $\left(\tilde{h}_{2}, \tilde{g}_{2}\right)$ be three HPFEs, $\lambda>0$. The hesitant Pythagorean fuzzy operation can be defined as

(1)

$$
\begin{aligned}
\tilde{f}_{1} \oplus \tilde{f}_{2} & =\underset{\mu_{1 k_{1}} \in \tilde{h}_{1}, v_{1 k_{1}} \in \tilde{g}_{1}, \mu_{2 k_{2}} \in \tilde{h}_{2}, \nu_{2 k_{2}} \in \tilde{g}_{2}}{\bigcup} \\
& \times\left\{\left(\sqrt{\mu_{1 k_{1}}^{2}+\mu_{2 k_{2}}^{2}-\mu_{1 k_{1}}^{2} \mu_{2 k_{2}}^{2}}, v_{1 k_{1}} v_{2 k_{2}}\right)\right\},
\end{aligned}
$$

(2)

$$
\begin{aligned}
& \tilde{f}_{1} \otimes \tilde{f}_{2}=\underset{\mu_{1 k_{1}} \in \tilde{h}_{1}, v_{1 k_{1}} \in \tilde{g}_{1}, \mu_{2 k_{2}} \in \tilde{h}_{2}, v_{2 k_{2}} \in \tilde{g}_{2}}{\bigcup} \\
& \quad \times\left\{\left(\mu_{1 k_{1}} \mu_{2 k_{2}}, \sqrt{v_{1 k_{1}}^{2}+v_{2 k_{2}}^{2}-v_{1 k_{1}}^{2} v_{2 k_{2}}^{2}}\right)\right\},
\end{aligned}
$$

(3) $\lambda \tilde{f}=\bigcup_{\mu_{k} \in \tilde{h}, v_{k} \in \tilde{g}}\left\{\left(\sqrt{1-\left(1-\mu_{k}^{2}\right)^{\lambda}},\left(v_{k}^{2}\right)^{\lambda}\right)\right\}$,
(4) $\tilde{f}^{\lambda}=\bigcup_{\mu_{k} \in \tilde{h}, \nu_{k} \in \tilde{g}}\left\{\left(\left(\mu_{k}\right)^{\lambda}, \sqrt{1-\left(1-v_{k}^{2}\right)^{\lambda}}\right)\right\}$.

In the following, we present hesitant Pythagorean fuzzy interaction operation laws as follows.

Definition 6 Let $\tilde{f}=(\tilde{h}, \tilde{g}), \tilde{f}_{1}=\left(\tilde{h}_{1}, \tilde{g}_{1}\right), \tilde{f}_{2}=\left(\tilde{h}_{2}, \tilde{g}_{2}\right)$ be three HPFEs, $\lambda>0$. The hesitant Pythagorean fuzzy interaction operations can be defined as

$$
\begin{aligned}
& \tilde{f}_{1} \oplus \tilde{f}_{2}=z \quad \bigcup \begin{array}{l}
\mu_{1 k_{1}} \in \tilde{h}_{1}, v_{1 k_{1}} \in \tilde{g}_{1}, \mu_{2 k_{2}} \in \tilde{h}_{2}, \nu_{2 k_{2}} \in \tilde{g}_{2} \\
\times
\end{array} \\
& \quad \times \sqrt{\mu_{1 k_{1}}^{2}+\mu_{2 k_{2}}^{2}-\mu_{1 k_{1}}^{2} \mu_{2 k_{2}}^{2}}, \\
&
\end{aligned}
$$

(2)

$$
\begin{aligned}
& \tilde{f}_{1} \otimes \tilde{f}_{2}=\underset{\mu_{1 k_{1}} \in \tilde{h}_{1}, v_{1 k_{1}} \in \tilde{g}_{1}, \mu_{2 k_{2}} \in \tilde{h}_{2}, v_{2 k_{2}} \in \tilde{g}_{2}}{ } \\
& \times\left\{\left(\left(\mu_{1 k_{1}}^{2}+\mu_{2 k_{2}}^{2}-\mu_{1 k_{1}}^{2} \mu_{2 k_{2}}^{2}\right.\right.\right. \\
& \left.\left.\left.-v_{1 k_{1}}^{2} \mu_{2 k_{2}}^{2}-\mu_{1 k_{1}}^{2} v_{2 k_{2}}^{2}\right)^{1 / 2}, \sqrt{v_{1 k_{1}}^{2}+v_{2 k_{2}}^{2}-v_{1 k_{1}}^{2} v_{2 k_{2}}^{2}}\right)\right\},
\end{aligned}
$$

(3)

$$
\begin{aligned}
\lambda \tilde{f}= & \bigcup_{\mu_{k} \in \tilde{h}, v_{k} \in \tilde{g}}\left\{\left(\sqrt{1-\left(1-\mu_{k}^{2}\right)^{\lambda}},\right.\right. \\
& \left.\left.\times \sqrt{\left(1-\mu_{k}^{2}\right)^{\lambda}-\left(1-\left(\mu_{k}^{2}+v_{k}^{2}\right)\right)^{\lambda}}\right)\right\},
\end{aligned}
$$

(4)

$$
\begin{aligned}
\tilde{f}^{\lambda}= & \bigcup_{\mu_{k} \in \tilde{h}, v_{k} \in \tilde{g}}\left\{\left(\sqrt{\left(1-v_{k}^{2}\right)^{\lambda}-\left(1-\left(\mu_{k}^{2}+v_{k}^{2}\right)\right)^{\lambda}},\right.\right. \\
& \left.\left.\times \sqrt{1-\left(1-v_{k}^{2}\right)^{\lambda}}\right)\right\} .
\end{aligned}
$$

The $\tilde{f}_{1} \oplus \tilde{f}_{2}$ and $\tilde{f}_{1} \otimes \tilde{f}_{2}$ can be rewritten as follows

$$
\begin{aligned}
& \tilde{f}_{1} \oplus \tilde{f}_{2}=\bigcup_{\mu_{1 k_{1}} \in \tilde{h}_{1}, v_{1 k_{1}} \in \tilde{g}_{1}, \mu_{2 k_{2}} \in \tilde{h}_{2}, \nu_{2 k_{2}} \in \tilde{g}_{2}} \\
& \times\left\{\left(\sqrt{1-\left(1-\mu_{1 k_{1}}^{2}\right)\left(1-\mu_{2 k_{2}}^{2}\right)},\right.\right. \\
& \left.\left.\times \sqrt{\left(1-\mu_{1 k_{1}}^{2}\right)\left(1-\mu_{2 k_{2}}^{2}\right)-\left(1-\left(\mu_{1 k_{1}}^{2}+v_{1 k_{1}}^{2}\right)\right)\left(1-\left(\mu_{2 k_{2}}^{2}+v_{2 k_{2}}^{2}\right)\right)}\right)\right\}, \\
& \tilde{f}_{1} \otimes \tilde{f}_{2}=\underset{\mu_{1 k_{1}} \in \tilde{h}_{1}, v_{1 k_{1}} \in \tilde{g}_{1}, \mu_{2 k_{2}} \in \tilde{h}_{2}, \nu_{2 k_{2}} \in \tilde{g}_{2}}{\bigcup}
\end{aligned}
$$




$$
\begin{aligned}
& \times\left\{\left(\left(\left(1-v_{1 k_{1}}^{2}\right)\left(1-v_{2 k_{2}}^{2}\right)-\left(1-\left(\mu_{1 k_{1}}^{2}+v_{1 k_{1}}^{2}\right)\right)\left(1-\left(\mu_{2 k_{2}}^{2}+v_{2 k_{2}}^{2}\right)\right)\right)^{1 / 2},\right.\right. \\
& \left.\left.\times \sqrt{1-\left(1-v_{1 k_{1}}^{2}\right)\left(1-v_{2 k_{2}}^{2}\right)}\right)\right\} .
\end{aligned}
$$

The results of above operations are still HPFEs. The proofs of (1) and (3) are given as follows and others can be proved similarly.

\section{Proof}

$\tilde{f}_{1} \oplus \tilde{f}_{2}=$

$$
\begin{aligned}
& \mu_{1 k_{1} \in \tilde{h}_{1}, v_{1 k_{1}} \in \tilde{g}_{1}, \mu_{2 k_{2}} \in \tilde{h}_{2}, v_{2 k_{2}} \in \tilde{g}_{2}} \\
\times & \times\left(\sqrt{\mu_{1 k_{1}}^{2}+\mu_{2 k_{2}}^{2}-\mu_{1 k_{1}}^{2} \mu_{2 k_{2}}^{2}},\right. \\
& \left.\left.\times \sqrt{v_{1 k_{1}}^{2}+v_{2 k_{2}}^{2}-v_{1 k_{1}}^{2} v_{2 k_{2}}^{2}-\mu_{1 k_{1}}^{2} v_{2 k_{2}}^{2}-v_{1 k_{1}}^{2} \mu_{2 k_{2}}^{2}}\right)\right\}, \\
& \left(\sqrt{\mu_{1 k_{1}}^{2}+\mu_{2 k_{2}}^{2}-\mu_{1 k_{1}}^{2} \mu_{2 k_{2}}^{2}}\right)^{2} \\
& +\left(\left(v_{1 k_{1}}^{2}+v_{2 k_{2}}^{2}-v_{1 k_{1}}^{2} v_{2 k_{2}}^{2}-\mu_{1 k_{1}}^{2} v_{2 k_{2}}^{2} v_{1 k_{1}}^{2} \mu_{2 k_{2}}^{2}\right)^{1 / 2}\right)^{2} \\
= & \mu_{1 k_{1}}^{2}+\mu_{2 k_{2}}^{2}-\mu_{1 k_{1}}^{2} \mu_{2 k_{2}}^{2}+v_{1 k_{1}}^{2}+v_{2 k_{2}}^{2} \\
& -v_{1 k_{1}}^{2} v_{2 k_{2}}^{2}-\mu_{1 k_{1}}^{2} v_{2 k_{2}}^{2}-v_{1 k_{1}}^{2} \mu_{2 k_{2}}^{2} \\
= & 1-\left(1-\mu_{1 k_{1}}^{2}\right)\left(1-\mu_{2 k_{2}}^{2}\right) \\
& +\left(1-\mu_{1 k_{1}}^{2}\right)\left(1-\mu_{2 k_{2}}^{2}\right)-\left(1-\left(\mu_{1 k_{1}}^{2}+v_{1 k_{1}}^{2}\right)\right) * \\
& \times\left(1-\left(\mu_{2 k_{2}}^{2}+v_{2 k_{2}}^{2}\right)\right) \\
= & 1-\left(1-\left(\mu_{1 k_{1}}^{2}+v_{1 k_{1}}^{2}\right)\right)\left(1-\left(\mu_{2 k_{2}}^{2}+v_{2 k_{2}}^{2}\right)\right) .
\end{aligned}
$$

Since $0 \leq \mu_{1 k_{1}}^{2}+v_{1 k_{1}}^{2} \leq 1,0 \leq \mu_{2 k_{2}}^{2}+v_{2 k_{2}}^{2} \leq 1$, then $0 \leq\left(1-\left(\mu_{1 k_{1}}^{2}+v_{1 k_{1}}^{2}\right)\right)\left(1-\left(\mu_{2 k_{2}}^{2}+v_{2 k_{2}}^{2}\right)\right) \leq 1$ and $0 \leq$ $1-\left(1-\left(\mu_{1 k_{1}}^{2}+v_{1 k_{1}}^{2}\right)\right)\left(1-\left(\mu_{2 k_{2}}^{2}+v_{2 k_{2}}^{2}\right)\right) \leq 1$. Hence, $\tilde{f}_{1} \oplus \tilde{f}_{2}$ is still an HPFE.

(3)

$$
\begin{aligned}
\lambda \tilde{f} & =\bigcup_{\mu_{k} \in \tilde{h}, v_{k} \in \tilde{g}} \\
& \times\left\{\left(\sqrt{1-\left(1-\mu_{k}^{2}\right)^{\lambda}},\right.\right. \\
& \left.\left.\times \sqrt{\left(1-\mu_{k}^{2}\right)^{\lambda}-\left(1-\left(\mu_{k}^{2}+v_{k}^{2}\right)\right)^{\lambda}}\right)\right\}, \\
& \left(\sqrt{1-\left(1-\mu_{k}^{2}\right)^{\lambda}}\right)^{2} \\
& +\left(\sqrt{\left(1-\mu_{k}^{2}\right)^{\lambda}-\left(1-\left(\mu_{k}^{2}+v_{k}^{2}\right)\right)^{\lambda}}\right)^{2} \\
= & 1-\left(1-\mu_{k}^{2}\right)^{\lambda}+\left(1-\mu_{k}^{2}\right)^{\lambda}-\left(1-\left(\mu_{k}^{2}+v_{k}^{2}\right)\right)^{\lambda} \\
= & 1-\left(1-\left(\mu_{k}^{2}+v_{k}^{2}\right)\right)^{\lambda} .
\end{aligned}
$$

Since $0 \leq \mu_{k}^{2}+v_{k}^{2} \leq 1,0 \leq\left(1-\left(\mu_{k}^{2}+v_{k}^{2}\right)\right)^{\lambda} \leq 1$, $0 \leq 1-\left(1-\left(\mu_{k}^{2}+v_{k}^{2}\right)\right)^{\lambda} \leq 1$. Then, the $\lambda \tilde{f}$ is still an HPFE.
Theorem 1 Let $\tilde{f}=(\tilde{h}, \tilde{g}), \tilde{f}_{1}=\left(\tilde{h}_{1}, \tilde{g}_{1}\right)$ and $\tilde{f}_{2}=\left(\tilde{h}_{2}, \tilde{g}_{2}\right)$ be three HPFEs, then we have

(1) $\tilde{f}_{1} \oplus \tilde{f}_{2}=\tilde{f}_{2} \oplus \tilde{f}_{1}$,

(2) $\tilde{f}_{1} \otimes \tilde{f}_{2}=\tilde{f}_{2} \otimes \tilde{f}_{1}$,

(3) $k\left(\tilde{f}_{1} \oplus \tilde{f}_{2}\right)=k \tilde{f}_{1} \oplus k \tilde{f}_{2}$,

(4) $k\left(\tilde{f}_{1} \otimes \tilde{f}_{2}\right)=k \tilde{f}_{1} \otimes k \tilde{f}_{2}$,

(5) $\left(k_{1}+k_{2}\right) \tilde{f}=k_{1} \tilde{f} \oplus k_{2} \tilde{f}$,

(6) $\tilde{f}^{k_{1}} \otimes \tilde{f}^{k_{2}}=\tilde{f}^{\left(k_{1}+k_{2}\right)}$.

Proof We only prove (1), (3) and (5), and others can be proved similarly.

$$
\begin{aligned}
& \tilde{f}_{1} \oplus \tilde{f}_{2}=\bigcup_{\mu_{1 k_{1} \in \tilde{h}_{1}, v_{1 k_{1}} \in \tilde{g}_{1}, \mu_{2 k_{2}} \in \tilde{h}_{2}, v_{2 k_{2}} \in \tilde{g}_{2}}} \\
& \quad \times\left\{\left(\sqrt{\mu_{1 k_{1}}^{2}+\mu_{2 k_{2}}^{2}-\mu_{1 k_{1}}^{2} \mu_{2 k_{2}}^{2}},\left(v_{1 k_{1}}^{2}\right.\right.\right. \\
& \left.\left.\left.+v_{2 k_{2}}^{2}-v_{1 k_{1}}^{2} v_{2 k_{2}}^{2}-\mu_{1 k_{1}}^{2} v_{2 k_{2}}^{2}-v_{1 k_{1}}^{2} \mu_{2 k_{2}}^{2}\right)^{1 / 2}\right)\right\} \\
& =\quad \mu_{\mu_{1 k_{1}} \in \tilde{h}_{1}, v_{1 k_{1}} \in \tilde{g}_{1}, \mu_{2 k_{2}} \in \tilde{h}_{2}, v_{2 k_{2}} \in \tilde{g}_{2}} \\
& \quad \times\left\{\left(\sqrt{\mu_{2 k_{2}}^{2}+\mu_{1 k_{1}}^{2}-\mu_{2 k_{2}}^{2} \mu_{1 k_{1}}^{2}},\left(v_{2 k_{2}}^{2}\right.\right.\right. \\
& \left.\left.\left.\quad+v_{1 k_{1}}^{2}-v_{2 k_{2}}^{2} v_{1 k_{1}}^{2}-\mu_{2 k_{2}}^{2} v_{1 k_{1}}^{2}-v_{2 k_{2}}^{2} \mu_{1 k_{1}}^{2}\right)^{1 / 2}\right)\right\} \\
& =\tilde{f}_{2} \oplus \tilde{f}_{1} .
\end{aligned}
$$

$$
\begin{aligned}
& \tilde{f}_{1} \oplus \tilde{f}_{2}= \\
& \mu_{1 k_{1}} \in \tilde{h}_{1}, v_{1 k_{1}} \in \tilde{g}_{1}, \mu_{2 k_{2}} \in \tilde{h}_{2}, v_{2 k_{2}} \in \tilde{g}_{2} \\
& \times\left\{\left(\sqrt{1-\left(1-\mu_{1 k_{1}}^{2}\right)\left(1-\mu_{2 k_{2}}^{2}\right)},\right.\right. \\
& \left.\left.\times \sqrt{\left(1-\mu_{1 k_{1}}^{2}\right)\left(1-\mu_{2 k_{2}}^{2}\right)-\left(1-\left(\mu_{1 k_{1}}^{2}+v_{1 k_{1}}^{2}\right)\right)\left(1-\left(\mu_{2 k_{2}}^{2}+v_{2 k_{2}}^{2}\right)\right)}\right)\right\} \\
& =\left\{\left(\mu_{\tilde{f}_{1} \oplus \tilde{f}_{2}}, v_{\tilde{f}_{1} \oplus \tilde{f}_{2}}\right)\right\} \text {. } \\
& k\left(\tilde{f}_{1} \oplus \tilde{f}_{2}\right)= \\
& \mu_{1 k_{1}} \in \tilde{h}_{1}, v_{1 k_{1}} \in \tilde{g}_{1}, \mu_{2 k_{2}} \in \tilde{h}_{2}, v_{2 k_{2}} \in \tilde{g}_{2} \\
& \times\left\{\left(\sqrt{1-\left(1-\mu_{1 k_{1}}^{2}\right)^{k}\left(1-\mu_{2 k_{2}}^{2}\right)^{k}},\right.\right. \\
& \left.\left.\times \sqrt{\left(1-\mu_{1 k_{1}}^{2}\right)^{k}\left(1-\mu_{2 k_{2}}^{2}\right)^{k}-\left(1-\left(\mu_{1 k_{1}}^{2}+\nu_{1 k_{1}}^{2}\right)\right)^{k}\left(1-\left(\mu_{2 k_{2}}^{2}+v_{2 k_{2}}^{2}\right)\right)^{k}}\right)\right\}, \\
& k \tilde{f}_{1}=\bigcup_{\mu_{1 k_{1}} \in \bar{h}_{1}, v_{1 k_{1}} \in \tilde{b}_{1}} \\
& \times\left\{\left(\sqrt{1-\left(1-\mu_{1 k_{1}}^{2}\right)^{k}}, \sqrt{\left(1-\mu_{1 k_{1}}^{2}\right)^{k}-\left(1-\left(\mu_{1 k_{1}}^{2}+\nu_{1 k_{1}}^{2}\right)\right)^{k}}\right)\right\} \\
& =\bigcup\left\{\left(\mu_{k \tilde{h}_{1}}, v_{k \tilde{h}_{1}}\right)\right\} \text {, } \\
& k \tilde{f}_{2}=\bigcup_{\mu_{2 k_{2}} \in \bar{h}_{2}, v_{2 k_{2}} \in \tilde{g}_{2}} \\
& \times\left\{\left(\sqrt{1-\left(1-\mu_{2 k_{2}}^{2}\right)^{k}},\right.\right.
\end{aligned}
$$




$$
\begin{aligned}
& \left.\left.\times \sqrt{\left(1-\mu_{2 k_{2}}^{2}\right)^{k}-\left(1-\left(\mu_{2 k_{2}}^{2}+v_{2 k_{2}}^{2}\right)\right)^{k}}\right)\right\} \\
= & \bigcup\left\{\left(\mu_{k \tilde{h}_{2}}, v_{k \tilde{h}_{2}}\right)\right\}, \\
k \tilde{f}_{1} \oplus k \tilde{f}_{2}= & \bigcup_{\mu_{1 k_{1}} \in \tilde{h}_{1}, v_{1 k_{1}} \in \tilde{g}_{1}, \mu_{2 k_{2}} \in \tilde{h}_{2}, v_{2 k_{2}} \in \tilde{g}_{2}} \\
& \times\left\{\left(\sqrt{1-\left(1-\mu_{1 k_{1}}\right)^{k}\left(1-\mu_{2 k_{2}}\right)^{k}},\left(\left(1-\mu_{1 k_{1}}\right)^{k}\left(1-\mu_{2 k_{2}}\right)^{k}-\left(1-\left(\mu_{1 k_{1}}^{2}\right.\right.\right.\right.\right. \\
& \left.\left.\left.\left.\left.+v_{1 k_{1}}^{2}\right)\right)^{k}\left(1-\left(\mu_{2 k_{2}}^{2}+v_{2 k_{2}}^{2}\right)\right)^{k}\right)^{1 / 2}\right)\right\} \\
= & k\left(\tilde{f}_{1} \oplus \tilde{f}_{2}\right) .
\end{aligned}
$$

$$
\begin{aligned}
& \left(k_{1}+k_{2}\right) \tilde{f}=\bigcup_{\mu_{k} \in \tilde{h}, v_{k} \in \tilde{g}} \\
& \times\left\{\left(\sqrt{1-\left(1-\mu_{k}^{2}\right)^{k_{1}+k_{2}}},\left(\left(1-\mu_{k}^{2}\right)^{k_{1}+k_{2}}\right.\right.\right. \\
& \left.\left.\left.-\left(1-\left(\mu_{k}^{2}+v_{k}^{2}\right)\right)^{k_{1}+k_{2}}\right)^{1 / 2}\right)\right\} \text {, } \\
& k_{1} \tilde{f}=\bigcup_{\mu_{k} \in \tilde{h}, v_{k} \in \tilde{g}}\left\{\left(\sqrt{1-\left(1-\mu_{k}^{2}\right)^{k_{1}}},\right.\right. \\
& \left.\left.\times \sqrt{\left(1-\mu_{k}^{2}\right)^{k_{1}}-\left(1-\left(\mu_{k}^{2}+v_{k}^{2}\right)\right)^{k_{1}}}\right)\right\}, \\
& k_{2} \tilde{f}=\bigcup_{\mu_{k} \in \tilde{h}, v_{k} \in \tilde{g}}\left\{\left(\sqrt{1-\left(1-\mu_{k}^{2}\right)^{k_{2}}},\right.\right. \\
& \left.\left.\times \sqrt{\left(1-\mu_{k}^{2}\right)^{k_{2}}-\left(1-\left(\mu_{k}^{2}+v_{k}^{2}\right)\right)^{k_{2}}}\right)\right\}, \\
& k_{1} \tilde{f} \oplus k_{2} \tilde{f}=\bigcup_{\mu_{k} \in \tilde{h}, v_{k} \in \tilde{g}}\left\{\left(\sqrt{1-\left(1-\mu_{k}\right)^{k_{1}}\left(1-\mu_{k}\right)^{k_{2}}}\right.\right. \text {, } \\
& \times\left(\left(1-\mu_{k}^{2}\right)^{k_{1}}\left(1-\mu_{k}^{2}\right)^{k_{2}}\right. \\
& \left.\left.\left.-\left(1-\left(\mu_{k}^{2}+v_{k}^{2}\right)\right)^{k_{1}}\left(1-\left(\mu_{k}^{2}+v_{k}^{2}\right)\right)^{k_{2}}\right)^{1 / 2}\right)\right\} \\
& =\bigcup_{\mu_{k} \in \tilde{h}, v_{k} \in \tilde{g}}\left\{\left(\sqrt{1-\left(1-\mu_{k}\right)^{k_{1}+k_{2}}}\right.\right. \text {, } \\
& \left.\left.\times\left(\left(1-\mu_{k}^{2}\right)^{k_{1}+k_{2}}-\left(1-\left(\mu_{k}^{2}+v_{k}^{2}\right)\right)^{k_{1}+k_{2}}\right)^{1 / 2}\right)\right\} \\
& =\left(k_{1}+k_{2}\right) \tilde{f} \text {. }
\end{aligned}
$$

Definition 7 Let $\tilde{f}=(\tilde{h}, \tilde{g})$ be an HPFE. The score of $\tilde{f}$ can be defined as

$S(\tilde{f})=\frac{1}{l_{\tilde{h}}} \sum_{\mu_{k} \in \tilde{h}} \mu_{k}^{2}-\frac{1}{l_{\tilde{g}}} \sum_{\nu_{k} \in \tilde{g}} v_{k}^{2}$.

The accuracy function can be defined as

$$
A(\tilde{f})=\frac{1}{l_{\tilde{h}}} \sum_{\mu_{k} \in \tilde{h}} \mu_{k}^{2}+\frac{1}{l_{\tilde{g}}} \sum_{v_{k} \in \tilde{g}} v_{k}^{2}
$$

where $l_{\tilde{h}}$ is the number of memberships in $\tilde{h}$ and $l_{\tilde{g}}$ is the number of non-memberships in $\tilde{g}$.

Definition 8 Let $\tilde{f}_{1}, \tilde{f}_{2}$ be two HPFEs. Then if

(1) If $S\left(\tilde{f}_{1}\right)>S\left(\tilde{f}_{2}\right)$, then $\tilde{f}_{1}>\tilde{f}_{2}$,

(2) If $S\left(\tilde{f}_{1}\right)=S\left(\tilde{f}_{2}\right)$, then

If $A\left(\tilde{f}_{1}\right)>A\left(\tilde{f}_{2}\right)$, then $\tilde{f}_{1}>\tilde{f}_{2}$,

If $A\left(\tilde{f}_{1}\right)=A\left(\tilde{f}_{2}\right)$, then $\tilde{f}_{1} \sim \tilde{f}_{2}$.

To define distance measure between HPFEs more accurately, the two HPFEs should have the same number of memberships and non-memberships. The HPFEs can be extended according to the risk attitude of decision-makers. If the decision-maker is risk seeking, the largest Pythagorean fuzzy value can be added; if decision-maker is risk averse, the smallest Pythagorean fuzzy value can be added; and if decision- maker is risk neutral, the average value of Pythagorean fuzzy values can be added.

Definition 9 The distance between two extended hesitant Pythagorean fuzzy values $\tilde{f}_{1}=\left(\tilde{h}_{1}, \tilde{g}_{1}\right)$ and $\tilde{f}_{2}=\left(\tilde{h}_{2}, \tilde{g}_{2}\right)$ can be defined as

$d\left(\tilde{f}_{1}, \tilde{f}_{2}\right)=\frac{1}{l_{\tilde{h}_{1}}} \sum\left(\left|\mu_{1 k_{1}}^{2}-\mu_{2 k_{2}}^{2}\right|+\left|v_{1 k_{1}}^{2}-v_{2 k_{2}}^{2}\right|\right) / 2$,

where $\mu_{1 k_{1}} \in \tilde{h}_{1}, v_{1 k_{1}} \in \tilde{g}_{1}, \mu_{2 k_{2}} \in \tilde{h}_{2}, v_{2 k_{2}} \in \tilde{g}_{2}$ and $l_{\tilde{h}_{1}}$ is the number of memberships in the $\tilde{f}_{1}$.

\section{Hesitant Pythagorean fuzzy interaction aggregation operators}

Definition 10 Let $\tilde{f}_{i}(i=1,2, \ldots, n)$ be a collection of HPFEs. The hesitant Pythagorean fuzzy interaction weighted averaging (HPFIWA) operator can be defined as

$\operatorname{HPFIWA}\left(\tilde{f}_{1}, \tilde{f}_{2}, \ldots, \tilde{f}_{n}\right)=\oplus_{j=1}^{n} w_{j} \tilde{f}_{j}$.

Theorem 2 Let $\tilde{f}_{i}=\left(\tilde{h}_{i}, \tilde{g}_{i}\right)=\bigcup_{\mu_{i k_{i}} \in \tilde{h}_{i}, v_{i k_{i}} \in \tilde{g}_{i}}\left\{\left(\mu_{i k_{i}}, v_{i k_{i}}\right)\right\}$ $(i=1,2, \ldots, n)$ be a collection of HPFEs. The aggregated value of the HPFIWA operator is still an HPFE, that is

$$
\begin{aligned}
& \operatorname{HPFIWA}\left(\tilde{f}_{1}, \tilde{f}_{2}, \ldots, \tilde{f}_{n}\right) \\
& =\bigcup_{\mu_{i k_{i}} \in \tilde{h}_{i}, v_{i k_{i}} \in \tilde{g}_{i}}\left\{\left(\sqrt{1-\prod_{i=1}^{n}\left(1-\mu_{i k_{i}}^{2}\right)^{w_{i}},}\right.\right. \\
& \quad \times\left(\prod_{i=1}^{n}\left(1-\mu_{i k_{i}}^{2}\right)^{w_{i}}\right. \\
& \left.\left.\left.\quad-\prod_{i=1}^{n}\left(1-\left(\mu_{i k_{i}}^{2}+v_{i k_{i}}^{2}\right)\right)^{w_{i}}\right)^{1 / 2}\right)\right\} .
\end{aligned}
$$


Proof The theorem can be proved using mathematical induction.

$$
\text { If } n=2, \operatorname{HPFIWA}\left(\tilde{f}_{1}, \tilde{f}_{2}\right)=w_{1} \tilde{f}_{1} \oplus w_{2} \tilde{f}_{2} .
$$

$$
\begin{aligned}
& w_{1} \tilde{f}_{1}=\bigcup_{\mu_{1 k_{1}} \in \tilde{h}_{1}, v_{1 k_{1}} \in \tilde{g}_{1}}\left\{\left(\sqrt{1-\left(1-\mu_{1 k_{1}}^{2}\right)^{w_{1}}},\left(\left(1-\mu_{1 k_{1}}^{2}\right)^{w_{1}}\right.\right.\right. \\
& \left.\left.\left.-\left(1-\left(\mu_{1 k_{1}}^{2}+v_{1 k_{1}}^{2}\right)\right)^{w_{1}}\right)^{1 / 2}\right)\right\},
\end{aligned}
$$

$w_{2} \tilde{f}_{2}=\bigcup_{\mu_{2 k_{2}} \in \tilde{h}_{2}, v_{2 k_{2}} \in \tilde{g}_{2}}\left\{\left(\sqrt{1-\left(1-\mu_{2 k_{2}}^{2}\right)^{w_{2}}},\left(\left(1-\mu_{2 k_{2}}^{2}\right)^{w_{2}}\right.\right.\right.$

$$
\left.\left.\left.-\left(1-\left(\mu_{2 k_{2}}^{2}+v_{2 k_{2}}^{2}\right)\right)^{w_{2}}\right)^{1 / 2}\right)\right\}
$$

$w_{1} \tilde{f}_{1} \oplus w_{2} \tilde{f}_{2}$

$$
\begin{aligned}
= & \bigcup \\
& \mu_{1 k_{1} \in \tilde{h}_{1}, v_{1 k_{1}} \in \tilde{g}_{1}, \mu_{2 k_{2}} \in \tilde{h}_{2}, v_{2 k_{2}} \in \tilde{g}_{2}} \\
& \times\left\{\left(\left(1-\left(1-\mu_{1 k_{1}}^{2}\right)^{w_{1}}\left(1-\mu_{2 k_{2}}^{2}\right)^{w_{2}}\right)^{1 / 2}\right.\right. \\
& \times\left(\left(1-\mu_{1 k_{1}}^{2}\right)^{w_{1}}\left(1-\mu_{2 k_{2}}^{2}\right)^{w_{2}}-\left(1-\left(\mu_{1 k_{1}}^{2}+v_{1 k}^{2}\right)\right)^{w_{1}}\right. \\
& \left.\left.\left.\left(1-\left(\mu_{2 k_{2}}^{2}+v_{2 k_{2}}^{2}\right)\right)^{w_{2}}\right)^{1 / 2}\right)\right\} \\
= & \bigcup_{\mu_{i k_{i}} \in \tilde{h}_{i}, v_{i k_{i}} \in \tilde{g}_{i}}\left\{\left(\left(1-\prod_{i=1}^{2}\left(1-\mu_{i k_{i}}^{2}\right)^{w_{i}}\right)^{1 / 2},\right.\right. \\
& \times\left(\prod_{i=1}^{2}\left(1-\mu_{i k_{i}}^{2}\right)^{w_{i}}\right. \\
& \left.\left.\left.-\prod_{i=1}^{2}\left(1-\left(\mu_{i k_{i}}^{2}+v_{i k_{i}}^{2}\right)\right)^{w_{i}}\right)^{1 / 2}\right)\right\} .
\end{aligned}
$$

Suppose Eq. (8) holds for $n=l$, that is

$\operatorname{HPFIWA}\left(\tilde{f}_{1}, \tilde{f}_{2}, \ldots, \tilde{f}_{l}\right)=\oplus_{i=1}^{l} w_{i} \tilde{f}_{i}$

$$
\begin{aligned}
= & \bigcup_{\mu_{i k_{i}} \in \tilde{h}_{i}, v_{i k_{i}} \in \tilde{g}_{i}}\left\{\left(\left(1-\prod_{i=1}^{l}\left(1-\mu_{i k_{i}}^{2}\right)^{w_{i}}\right)^{1 / 2},\right.\right. \\
& \times\left(\prod_{i=1}^{l}\left(1-\mu_{i k_{i}}^{2}\right)^{w_{i}}\right. \\
& \left.\left.\left.-\prod_{i=1}^{l}\left(1-\left(\mu_{i k_{i}}^{2}+v_{i k_{i}}^{2}\right)\right)^{w_{i}}\right)^{1 / 2}\right)\right\} .
\end{aligned}
$$

If $n=l+1$, using the interaction operation laws of hesitant Pythagorean fuzzy value, we can get

$\operatorname{HPFIWA}\left(\tilde{f}_{1}, \tilde{f}_{2}, \ldots, \tilde{f}_{l}, \tilde{f}_{l+1}\right)=\left(\oplus_{j=1}^{l} w_{j} \tilde{f}_{j}\right) \oplus\left(w_{l+1}\right.$ $\left.\tilde{f}_{l+1}\right)$,

$$
\begin{aligned}
& w_{l+1} \tilde{f}_{l+1}=\bigcup_{\mu_{l+1 k_{l+1}} \in \tilde{h}_{l+1}, \nu_{l+1 k_{l+1}} \in \tilde{g}_{l+1}}^{2} \\
& \times\left(\left(1-\left(1-\mu_{l+1 k_{l+1}}\right)^{w_{l+1}}\right)^{1 / 2},\left(\left(1-\mu_{l+1 k_{l+1}}^{2}\right)^{w_{l+1}}\right.\right. \\
& \left.\left.-\left(1-\left(\mu_{l+1 k_{l+1}}^{2}+v_{l+1 k_{l+1}}^{2}\right)\right)^{w_{l+1}}\right)^{1 / 2}\right),
\end{aligned}
$$

$\operatorname{HPFIWA}\left(\tilde{f}_{1}, \tilde{f}_{2}, \ldots, \tilde{f}_{l}, \tilde{f}_{l+1}\right)$

$$
\begin{aligned}
& =\left(\oplus_{j=1}^{l} w_{j} \tilde{f}_{j}\right) \oplus\left(w_{l+1} \tilde{f}_{l+1}\right) \\
& =\bigcup \\
& \mu_{i k_{i}} \in \tilde{h}_{i}, v_{i k_{i}} \in \tilde{g}_{i} \\
& \times\left\{\left(\sqrt{1-\prod_{i=1}^{l}\left(1-\mu_{i k_{i}}^{2}\right)^{w_{i}}},\left(\prod_{i=1}^{l}\left(1-\mu_{i k_{i}}^{2}\right)^{w_{i}}\right.\right.\right. \\
& \left.\left.\left.-\prod_{i=1}^{l}\left(1-\left(\mu_{i k_{i}}^{2}+v_{i k_{i}}^{2}\right)\right)^{w_{i}}\right)^{1 / 2}\right)\right\} \oplus \\
& \mu_{l+1 k_{k+1}} \in \tilde{h}_{l+1}, \nu_{l+1 k_{l+1}} \in \tilde{g}_{l+1} \\
& \times\left\{\left(\sqrt{1-\left(1-\mu_{l+1 k_{l+1}}^{2}\right)^{w_{l+1}}},\left(\left(1-\mu_{l+1 k_{l+1}}^{2}\right)^{w_{l+1}}\right.\right.\right. \\
& \left.\left.\left.-\left(1-\left(\mu_{l+1 k_{l+1}}^{2}+v_{l+1 k_{l+1}}^{2}\right)\right)^{w_{l+1}}\right)^{1 / 2}\right)\right\} \\
& =\bigcup \\
& \mu_{i k_{i}} \in \tilde{h}_{i}, v_{i k_{i}} \in \tilde{g}_{i} \\
& \times\left\{\left(\sqrt{1-\prod_{i=1}^{l}\left(1-\mu_{i k_{i}}^{2}\right)^{w_{i}}\left(1-\mu_{l+1 k_{l+1}}^{2}\right)^{w_{l+1}},},\right.\right. \\
& \times\left(\prod_{i=1}^{l}\left(1-\mu_{i k_{i}}^{2}\right)^{w_{i}}\left(1-\mu_{l+1 k_{l+1}}^{2}\right)^{w_{l+1}}\right. \\
& -\prod_{i=1}^{l}\left(1-\left(\mu_{i k_{i}}^{2}+v_{i k_{i}}^{2}\right)\right)^{w_{i}} * \\
& \left.\left.\left.\times\left(1-\left(\mu_{l+1 k_{l+1}}^{2}+v_{l+1 k_{l+1}}^{2}\right)\right)^{w_{l+1}}\right)^{1 / 2}\right)\right\} \\
& =\bigcup_{\mu_{i k_{i}} \in \tilde{h}_{i}, v_{i k_{i}} \in \tilde{g}_{i}} \\
& \times\left\{\left(\sqrt{1-\prod_{i=1}^{l+1}\left(1-\mu_{i k_{i}}^{2}\right)^{w_{i}}},\left(\prod_{i=1}^{l+1}\left(1-\mu_{i k_{i}}^{2}\right)^{w_{i}}\right.\right.\right. \\
& \left.\left.\left.-\prod_{i=1}^{l+1}\left(1-\left(\mu_{i k_{i}}^{2}+v_{i k_{i}}^{2}\right)\right)^{w_{i}}\right)^{1 / 2}\right)\right\} \text {. }
\end{aligned}
$$

By mathematical induction, Eq. (8) holds for all $n$. Moreover, for each $(\mu, \nu)$ in the HPFIWA operator, we have $\mu^{2}+v^{2}=1-\prod_{i=1}^{n}\left(1-\left(\mu_{i k_{i}}^{2}+v_{i k_{i}}^{2}\right)\right)^{w_{i}}$. Since $0 \leq$ $\mu_{i k_{i}}^{2}+v_{i k_{i}}^{2} \leq 1$, we have $0 \leq \mu^{2}+v^{2} \leq 1$. Then, the aggregated result of the HPFIWA operator is still an HPFE.

If the weight vector is taken as $\left(\frac{1}{n}, \frac{1}{n}, \ldots, \frac{1}{n}\right)$, the HPFIWA operator reduces to the hesitant Pythagorean fuzzy interaction averaging (HPFIA) operator as follows

$$
\begin{aligned}
& \operatorname{HPFIA}\left(\tilde{f}_{1}, \tilde{f}_{2}, \ldots, \tilde{f}_{n}\right)=\frac{1}{n} \oplus_{j=1}^{n} \tilde{f}_{j} \\
& =\bigcup_{\mu_{i k_{i}} \in \tilde{h}_{i}, v_{i k_{i}} \in \tilde{g}_{i}}\left\{\left(\sqrt{1-\prod_{i=1}^{n}\left(1-\mu_{i k_{i}}^{2}\right)^{\frac{1}{n}}},\right.\right. \\
& \left.\left.\quad \times\left(\prod_{i=1}^{n}\left(1-\mu_{i k_{i}}^{2}\right)^{\frac{1}{n}}-\prod_{i=1}^{n}\left(1-\left(\mu_{i k_{i}}^{2}+v_{i k_{i}}^{2}\right)\right)^{\frac{1}{n}}\right)^{1 / 2}\right)\right\} .
\end{aligned}
$$


Theorem 3 Let $\tilde{f}_{i}=\left(\tilde{h}_{i}, \tilde{g}_{i}\right)(i=1,2, \ldots, n)$ be a collection of HPFEs. If all the HPFEs reduces to $\tilde{f}=(\tilde{h}, \tilde{g})$, the HPFIWA operator reduces to the following form

$\operatorname{HPFIWA}\left(\tilde{f}_{1}, \tilde{f}_{2}, \ldots, \tilde{f}_{n}\right)=\tilde{f}$.

Theorem 4 Let $\tilde{f}_{i}=\left(\tilde{h}_{i}, \tilde{g}_{i}\right)(i=1,2, \ldots, n)$ be a collection of HPFEs. Let $\tilde{f}^{+}=(1,0), \tilde{f}^{-}=(0,1)$, then

$\tilde{f}^{-} \leq \operatorname{HPFIWA}\left(\tilde{f}_{1}, \tilde{f}_{2}, \ldots, \tilde{f}_{n}\right) \leq \tilde{f}^{+}$.

Example 1 Let $\tilde{f}_{1}=\{(0.9,0.0)\}, \tilde{f}_{2}=\{(0.6,0.5)\}, \tilde{f}_{3}=$ $\{(0.8,0.3),(0.7,0.4)\}$ and $W=(0.25,0.35,0.40)$. Using the HPFIWA operator, we can get $\operatorname{HPFIWA}\left(\tilde{f}_{1}, \tilde{f}_{2}, \tilde{f}_{3}\right)=$ $\{(0.7904,0.5649),(0.7541,0.5969)\}$.

Definition 11 Let $\tilde{f}_{i}(i=1,2, \ldots, n)$ be a collection of HPFEs. The hesitant Pythagorean fuzzy interaction weighted geometric averaging (HPFIWGA) operator can be defined as

$\operatorname{HPFIWGA}\left(\tilde{f}_{1}, \tilde{f}_{2}, \ldots, \tilde{f}_{n}\right)=\otimes_{j=1}^{n} \tilde{f}_{j}^{w_{j}}$.

Theorem 5 Let $\tilde{f}_{i}=\left(\tilde{h}_{i}, \tilde{g}_{i}\right)(i=1,2, \ldots, n)$ be a collection of HPFEs. Then, the aggregated result of the HPFIWGA operator is still an HPFE, which has the following form

$$
\begin{aligned}
& \operatorname{HPFIWGA}\left(\tilde{f}_{1}, \tilde{f}_{2}, \ldots, \tilde{f}_{n}\right) \\
& =\bigcup_{\mu_{i k_{i}} \in \tilde{h}_{i}, v_{i k_{i}} \in \tilde{g}_{i}} \\
& \quad \times\left\{\left(\sqrt{\prod_{i=1}^{n}\left(1-v_{i k_{i}}^{2}\right)^{w_{i}}-\prod_{i=1}^{n}\left(1-\left(\mu_{i k_{i}}^{2}+v_{i k_{i}}^{2}\right)\right)^{w_{i}}}\right.\right. \\
& \left.\left.\quad \times \sqrt{1-\prod_{i=1}^{n}\left(1-v_{i k_{i}}^{2}\right)^{w_{i}}}\right)\right\} .
\end{aligned}
$$

Proof If $n=2, \operatorname{HPFIWGA}\left(\tilde{f}_{1}, \tilde{f}_{2}\right)=\tilde{f}_{1}^{w_{1}} \otimes \tilde{f}_{2}^{w_{2}}$.

$$
\begin{aligned}
\tilde{f}_{1}^{w_{1}} & =\bigcup_{\mu_{1 k_{1}} \in \tilde{h}_{1}, v_{1 k_{1}} \in \tilde{g}_{1}} \\
& \times\left\{\left(\sqrt{\left(1-v_{1 k_{1}}^{2}\right)^{w_{1}}-\left(1-\left(\mu_{1 k_{1}}^{2}+v_{1 k_{1}}^{2}\right)\right)^{w_{1}}},\right.\right. \\
& \left.\left.\times \sqrt{1-\left(1-v_{1 k_{1}}^{2}\right)^{w_{1}}}\right)\right\}, \\
\tilde{f}_{2}^{w_{2}} & =\bigcup \\
& \times\left\{\left(\sqrt{\left(1-v_{2 k_{2}}^{2}\right)^{w_{2}}-\left(1-\left(\mu_{2 k_{2}}^{2}+v_{2 k_{2}}^{2}\right)\right)^{w_{2}}},\right.\right. \\
& \left.\left.\times \sqrt{1-\left(1-v_{2 k_{2}}^{2}\right)^{w_{2}}}\right)\right\} .
\end{aligned}
$$

$$
\begin{aligned}
\tilde{f}_{1}^{w_{1}} \otimes & \tilde{f}_{2}^{w_{2}} \\
= & \bigcup_{\mu_{i k_{i}} \in \tilde{h}_{i}, v_{i k_{i}} \in \tilde{g}_{i}} \\
& \times\left\{\left(\left(\left(1-v_{1 k_{1}}^{2}\right)^{w_{1}}\left(1-v_{2 k_{2}}^{2}\right)^{w_{2}}-\left(1-\left(\mu_{1 k_{1}}^{2}+v_{1 k_{2}}^{2}\right)\right)^{w_{1}}\right.\right.\right. \\
& \left.\times\left(1-\left(\mu_{2 k_{2}}^{2}+v_{2 k_{2}}^{2}\right)\right)^{w_{2}}\right)^{1 / 2}, \\
& \left.\left.\times \sqrt{1-\left(1-v_{1 k_{1}}^{2}\right)^{w_{1}}\left(1-v_{2 k_{2}}^{2}\right)^{w_{2}}}\right)\right\} \\
= & \mu_{i k_{i} \in \tilde{h}_{i}, v_{i k_{i}} \in \tilde{g}_{i}} \\
& \times\left\{\left(\sqrt{\prod_{i=1}^{2}\left(1-v_{i k_{i}}^{2}\right)^{w_{i}}-\prod_{i=1}^{2}\left(1-\left(\mu_{i k_{i}}^{2}+v_{i k_{i}}^{2}\right)\right)^{w_{i}}},\right.\right. \\
& \left.\left.\times \sqrt{1-\prod_{i=1}^{2}\left(1-v_{i k_{i}}^{2}\right)^{w_{i}}}\right)\right\} .
\end{aligned}
$$

Equation (10) holds for $n=2$. If Eq. (10) is established for $n=l$, i.e.

$$
\begin{aligned}
& \otimes_{i=1}^{l} \tilde{f}_{i}^{w_{i}}=\bigcup_{\mu_{i k_{i}} \in \tilde{h}_{i}, v_{i k_{i}} \in \tilde{g}_{i}} \\
& \times\left\{\left(\left(\prod_{i=1}^{l}\left(1-v_{i k_{i}}^{2}\right)^{w_{i}}-\prod_{i=1}^{l}\left(1-\left(\mu_{i k_{i}}^{2}+v_{i k_{i}}^{2}\right)\right)^{w_{i}}\right)^{1 / 2},\right.\right. \\
& \left.\left.\times \sqrt{1-\prod_{i=1}^{l}\left(1-v_{i k_{i}}^{2}\right)^{w_{i}}}\right)\right\} .
\end{aligned}
$$

Then for $n=l+1, \otimes_{i=1}^{l+1} \tilde{f}_{i}^{w_{i}}=\left(\otimes_{i=1}^{l} \tilde{f}_{i}^{w_{i}}\right) \otimes\left(\tilde{f}_{l+1}^{w_{l+1}}\right)$. By Eq.(10), we have

$$
\begin{aligned}
& \tilde{f}_{l+1}^{w_{l+1}}=\bigcup_{\mu_{l+1 k_{l+1}} \in \tilde{h}_{l+1}, v_{l+1 l_{l+1}} \in \tilde{g}_{l+1}} \\
& \times\left\{\left(\left(\left(1-v_{l+1 k_{l+1}}^{2}\right)^{w_{l+1}}\right.\right.\right. \\
& \left.-\left(1-\left(\mu_{l+1 k_{l+1}}^{2}+v_{l+1 k_{l+1}}^{2}\right)\right)^{w_{l+1}}\right)^{1 / 2}, \\
& \left.\left.\times \sqrt{1-\left(1-v_{l+1 k_{l+1}}^{2}\right)^{w_{l+1}}}\right)\right\} \text {. } \\
& \otimes_{i=1}^{l+1} \tilde{f}_{i}^{w_{i}}=\left(\otimes_{i=1}^{l} \tilde{f}_{i}^{w_{i}}\right) \otimes\left(\tilde{f}_{l+1}^{w_{l+1}}\right) \\
& =\bigcup \\
& \mu_{i k_{i}} \in \tilde{h}_{i}, v_{i k_{i}} \in \tilde{g}_{i} \\
& \times\left\{\left(\left(1-\left(1-\prod_{i=1}^{l}\left(1-v_{i k_{i}}^{2}\right)^{w_{i}}\right)\right)\right.\right. \\
& \times\left(1-\left(1-\left(1-v_{l+1 k_{l+1}}^{2}\right)^{w_{l+1} k_{l+1}}\right)\right) \\
& \times-\left(1-\left(1-\prod_{i=1}^{l}\left(1-\left(\mu_{i k_{i}}^{2}+v_{i k_{i}}^{2}\right)\right)^{w_{i}}\right)\right) \\
& \times\left(1-\left(1-\left(1-\left(\mu_{l+1 k_{l+1}}^{2}+v_{l+1 k_{l+1}}^{2}\right)\right)^{w_{l+1}}\right)\right)^{1 / 2} \text {, }
\end{aligned}
$$




$$
\begin{aligned}
& \times\left(1-\left(1-\left(1-\prod_{i=1}^{l}\left(1-v_{i k_{i}}^{2}\right)^{w_{i}}\right)\right) *\right. \\
& \left.\left.\left.\times\left(1-\left(1-\left(1-v_{l+1 k_{l+1}}^{2}\right)^{w_{k+1}}\right)\right)\right)^{1 / 2}\right)\right\} \\
& =\bigcup_{\mu_{i k_{i}} \in \tilde{h}_{i}, v_{i k_{i}} \in \tilde{g}_{i}}\left\{\left(\left(\prod_{i=1}^{l}\left(1-v_{i k_{i}}^{2}\right)^{w_{i}}\left(1-v_{l+1 k_{l+1}}^{2}\right)^{w_{l+1}}\right.\right.\right. \\
& -\prod_{i=1}^{l}\left(1-\left(\mu_{i k_{i}}^{2}+v_{i k_{i}}^{2}\right)\right)^{w_{i}} \\
& \left.\left(1-\left(\mu_{l+1 k_{l+1}}^{2}+v_{l+1 k_{k+1}}^{2}\right)\right)^{w_{l+1}}\right)^{1 / 2} \text {, } \\
& \left.\left.\times\left(1-\prod_{i=1}^{k}\left(1-v_{i}^{2}\right)^{w_{i}} *\left(1-v_{k+1}^{2}\right)^{w_{k+1}}\right)^{1 / 2}\right)\right\} \\
& =\bigcup_{\mu_{i k_{i}} \in \tilde{h}_{i}, v_{i k_{i}} \in \tilde{g}_{i}}\left\{\left(\left(\prod_{i=1}^{l+1}\left(1-v_{i k_{i}}^{2}\right)^{w_{i}}\right.\right.\right. \\
& \left.-\prod_{i=1}^{l+1}\left(1-\left(\mu_{i k_{i}}^{2}+v_{i k_{i}}^{2}\right)\right)^{w_{i}}\right)^{1 / 2} \text {, } \\
& \left.\left.\times \sqrt{1-\prod_{i=1}^{k+1}\left(1-v_{i k_{i}}^{2}\right)^{w_{i}}}\right)\right\} \text {. }
\end{aligned}
$$

Then, Eq. (10) holds for $n=k+1$. Therefore, using mathematical induction on $n$, Eq. (10) holds for all $n$. Moreover, for each $(\mu, v)$ in the HPFIWGA operator, $\mu^{2}+v^{2}=$ $1-\prod_{i=1}^{l+1}\left(1-\left(\mu_{i k_{i}}^{2}+v_{i k_{i}}^{2}\right)\right)^{w_{i}}$. Since $0 \leq \mu_{i k_{i}}^{2}+v_{i k_{i}}^{2} \leq 1$, hence $0 \leq \mu^{2}+v^{2} \leq 1$. The aggregated result of the HPFIWGA operator is still an HPFE.

Theorem 6 Let $\tilde{f}_{i}=\left(\tilde{h}_{i}, \tilde{g}_{i}\right)(i=1,2, \ldots, n)$ be a collection of HPFEs. If all the HPFEs reduces to $\tilde{f}=(\tilde{h}, \tilde{g})$, the HPFIWGA operator reduces to the following form

$\operatorname{HPFIWGA}\left(\tilde{f}_{1}, \tilde{f}_{2}, \ldots, \tilde{f}_{n}\right)=\tilde{f}$.

Theorem 7 Let $\tilde{f}_{i}=\left(\tilde{h}_{i}, \tilde{g}_{i}\right)(i=1,2, \ldots, n)$ be a collection of HPFEs. Let $\tilde{f}^{+}=(1,0), \tilde{f}^{-}=(0,1)$, then

$$
\tilde{f}^{-} \leq \operatorname{HPFIWGA}\left(\tilde{f}_{1}, \tilde{f}_{2}, \ldots, \tilde{f}_{n}\right) \leq \tilde{f}^{+}
$$

If the weight vector is taken as $\left(\frac{1}{n}, \frac{1}{n}, \ldots, \frac{1}{n}\right)$, the HPFIWGA operator reduces to the hesitant Pythagorean fuzzy interaction geometric averaging (HPFIGA) operator as follows

$$
\begin{aligned}
& \operatorname{HPFIGA}\left(\tilde{f}_{1}, \tilde{f}_{2}, \ldots, \tilde{f}_{n}\right)=\otimes_{j=1}^{n} \tilde{f}_{j}^{\frac{1}{n}} \\
& =\bigcup_{\mu_{i k_{i}} \in \tilde{h}_{i}, v_{i k_{i}} \in \tilde{g}_{i}} \\
& \quad \times\left\{\left(\sqrt{\prod_{i=1}^{n}\left(1-v_{i k_{i}}^{2}\right)^{\frac{1}{n}}-\prod_{i=1}^{n}\left(1-\left(\mu_{i k_{i}}^{2}+v_{i k_{i}}^{2}\right)\right)^{\frac{1}{n}}},\right.\right.
\end{aligned}
$$

$$
\left.\left.\times \sqrt{1-\prod_{i=1}^{n}\left(1-v_{i k_{i}}^{2}\right)^{\frac{1}{n}}}\right)\right\} .
$$

Example 2 Suppose the Pythagorean fuzzy values are the same as that in Example 1. Using the HPFIWGA operator, we can get $\operatorname{HPFIWGA}\left(\tilde{f}_{1}, \tilde{f}_{2}, \tilde{f}_{3}\right)=\{(0.7678,0.3595)$, $(0.7289,0.3959)\}$.

Definition 12 Let $\tilde{f}_{i}=\left(\tilde{h}_{i}, \tilde{g}_{i}\right)(i=1,2, \ldots, n)$ be a collection of HPFEs. The generalized hesitant Pythagorean fuzzy interaction weighted averaging (GHPFIWA) operator can be defined as

$\operatorname{GHPFIWA}\left(\tilde{f}_{1}, \tilde{f}_{2}, \ldots, \tilde{f}_{n}\right)=\left(\oplus_{j=1}^{n} w_{j} \tilde{f}_{j}^{\lambda}\right)^{1 / \lambda}$.

Theorem 8 Let $\tilde{f}_{i}(i=1,2, \ldots, n)$ be a collection of HPFEs. Then

$$
\begin{aligned}
& \operatorname{GHPFI}_{\lambda}\left(\tilde{f}_{1}, \tilde{f}_{2}, \ldots, \tilde{f}_{n}\right)=\bigcup_{\mu_{i k_{i}} \in \tilde{h}_{i}, v_{i k_{i} \in \tilde{g}_{i}}} \\
& \times\left\{\left(\left(1-\left(\prod _ { i = 1 } ^ { n } \left(1-\left(\left(1-v_{i k_{i}}^{2}\right)^{\lambda}\right.\right.\right.\right.\right.\right. \\
& \left.\left.\left.-\left(1-\left(\mu_{i k_{i}}^{2}+v_{i k_{i}}^{2}\right)\right)^{\lambda}\right)^{w_{i}}-\prod_{i=1}^{n}\left(1-\left(\mu_{i k_{i}}^{2}+v_{i k_{i}}^{2}\right)\right)^{\lambda w_{i}}\right)\right)^{1 / \lambda} \\
& -\left(\prod_{i=1}^{n}\left(1-\left(\mu_{i k_{i}}^{2}+v_{i k_{i}}^{2}\right)\right)^{w_{i}}\right)^{1 / 2},\left(1-\left(1-\left(\prod_{i=1}^{n}(1-\right.\right.\right. \\
& \times\left(\left(1-v_{i k_{i k_{i}}}^{2}\right)^{\lambda}-\left(1-\left(\mu_{i k_{i}}^{2}+v_{i k_{i}}^{2}\right)\right)^{\lambda}\right)^{w_{i}} \\
& \left.\left.\left.\left.\left.\quad-\prod_{i=1}^{n}\left(1-\left(\mu_{i k_{i}}^{2}+v_{i k_{i}}^{2}\right)\right)^{\lambda w_{j}}\right)\right)^{1 / \lambda}\right)^{1 / 2}\right)\right\} .
\end{aligned}
$$

Proof If $n=2, \operatorname{GHPFIWA}_{\lambda}\left(\tilde{f}_{1}, \tilde{f}_{2}\right)=\left(w_{1} \tilde{f}_{1}^{\lambda} \oplus w_{2} \tilde{f}_{2}^{\lambda}\right)^{1 / \lambda}$,

$$
\begin{aligned}
& \tilde{f}_{1}^{w_{1}}=\bigcup \bigcup \\
& \mu_{1 k_{1}} \in \tilde{h}_{1}, v_{1 k_{1}} \in \tilde{g}_{1} \\
& \times\left\{\left(\sqrt{\left(1-v_{1 k_{1}}^{2}\right)^{w_{1}}-\left(1-\left(\mu_{1 k_{1}}^{2}+v_{1 k_{1}}^{2}\right)\right)^{w_{1}}},\right.\right. \\
& \left.\left.\times \sqrt{1-\left(1-v_{1 k_{1}}^{2}\right)^{w_{1}}}\right)\right\} \text {, } \\
& \tilde{f}_{2}^{w_{2}}=\bigcup_{\mu_{2 k_{2}} \in \tilde{h}_{2}, \nu_{2 k_{2}} \in \tilde{g}_{2}} \\
& \times\left\{\left(\sqrt{\left(1-v_{2 k_{2}}^{2}\right)^{w_{2}}-\left(1-\left(\mu_{2 k_{2}}^{2}+v_{2 k_{2}}^{2}\right)\right)^{w_{2}}},\right.\right. \\
& \left.\left.\times \sqrt{1-\left(1-v_{2 k_{2}}^{2}\right)^{w_{2}}}\right)\right\} \text {. } \\
& \tilde{f}_{1}^{w_{1}} \otimes \tilde{f}_{2}^{w_{2}} \\
& =\bigcup \\
& \mu_{i k_{i}} \in \tilde{h}_{i}, v_{i k_{i}} \in \tilde{g}_{i} \\
& \times\left\{\left(\left(\left(1-v_{1 k_{1}}^{2}\right)^{w_{1}}\left(1-v_{2 k_{2}}^{2}\right)^{w_{2}}-\left(1-\left(\mu_{1 k_{1}}^{2}\right.\right.\right.\right.\right.
\end{aligned}
$$




$$
\begin{aligned}
& \left.\left.\left.+v_{1 k_{1}}^{2}\right)\right)^{w_{1}}\left(1-\left(\mu_{2 k_{2}}^{2}+v_{2 k_{2}}^{2}\right)\right)^{w_{2}}\right)^{1 / 2}, \\
& \left.\left.\times \sqrt{1-\left(1-v_{1 k_{1}}^{2}\right)^{w_{1}}\left(1-v_{2 k_{2}}^{2}\right)^{w_{2}}}\right)\right\} \\
& =\bigcup \\
& \mu_{i k_{i}} \in \tilde{h}_{i}, v_{i k_{i}} \in \tilde{g}_{i} \\
& \times\left\{\left(\sqrt{\prod_{i=1}^{2}\left(1-v_{i k_{i}}^{2}\right)^{w_{i}}-\prod_{i=1}^{2}\left(1-\left(\mu_{i k_{i}}^{2}+v_{i k_{i}}^{2}\right)\right)^{w_{i}}},\right.\right. \\
& \left.\left.\times \sqrt{1-\prod_{i=1}^{2}\left(1-v_{i k_{i}}^{2}\right)^{w_{i}}}\right)\right\} \text {. }
\end{aligned}
$$

Hence, Eq. (12) holds for $n=2$. If Eq. (12) is established for $n=l$, i.e.

$$
\begin{aligned}
& \left(\oplus_{i=1}^{l} w_{i} \tilde{f}_{i}^{\lambda}\right)^{1 / \lambda}=\bigcup_{\mu_{i k_{i}} \in \tilde{h}_{i}, v_{i k_{i} \in \tilde{g}_{i}}} \\
& \quad \times\left\{\left(\left(1-\left(\prod _ { i = 1 } ^ { l } \left(1-\left(\left(1-v_{i k_{i}}^{2}\right)^{\lambda}\right.\right.\right.\right.\right.\right. \\
& \left.-\left(1-\left(\mu_{i k_{i}}^{2}+v_{i k_{i}}^{2}\right)\right)^{\lambda}\right)^{w_{i}} \\
& \left.\left.\quad-\prod_{i=1}^{l}\left(1-\left(\mu_{i k_{i}}^{2}+v_{i k_{i}}^{2}\right)\right)^{\lambda w_{i}}\right)\right)^{1 / \lambda} \\
& \left.-\prod_{i=1}^{l}\left(1-\left(\mu_{i k_{i}}^{2}+v_{i k_{i}}^{2}\right)\right)^{w_{i}}\right)^{1 / 2}, \\
& \times\left(1-\left(1-\left(\prod _ { i = 1 } ^ { l } \left(1-\left(\left(1-v_{i k_{i}}^{2}\right)^{\lambda}-\left(1-\left(\mu_{i k_{i}}^{2}+v_{i k_{i}}^{2}\right)\right)^{\lambda}\right)^{w_{i}}\right.\right.\right.\right. \\
& \left.\left.\left.\left.\left.\left.\quad \times-\prod_{i=1}^{l}\left(1-\left(\mu_{i k_{i}}^{2}+v_{i k_{i}}^{2}\right)\right)^{\lambda w_{i}}\right)\right)^{1 / \lambda}\right)\right)^{1 / 2}\right)\right\} .
\end{aligned}
$$

Then $n=l+1$,

$$
\begin{aligned}
& \left(\oplus_{i=1}^{l+1} w_{i} \tilde{f}_{i}^{\lambda}\right)^{1 / \lambda} \\
& =\bigcup_{\mu_{i k_{i}} \in \tilde{h}_{i}, v_{i k_{i}} \in \tilde{g}_{i}}\left(\left(\oplus_{i=1}^{l} w_{i} \tilde{f}_{i}^{\lambda}\right) \oplus\left(w_{l+1} \tilde{f}_{l+1}^{\lambda}\right)\right)^{1 / \lambda} \\
& \quad \tilde{f}_{l+1}^{\lambda}=\bigcup_{\mu_{i k_{i}} \in \tilde{h}_{i}, v_{i k_{i}} \in \tilde{g}_{i}} \\
& \quad \times\left\{\left(\left(\left(1-v_{l+1 k_{l+1}}^{2}\right)^{\lambda}-\left(1-\left(\mu_{l+1 k_{l+1}}^{2}\right.\right.\right.\right.\right. \\
& \left.\left.\left.\left.\left.\quad+v_{l+1 k_{l+1}}^{2}\right)\right)^{\lambda}\right)^{1 / 2}, \sqrt{1-\left(1-v_{l+1 k_{l+1}}^{2}\right)^{\lambda}}\right)\right\}, \\
& w_{l+1} \tilde{f}_{l+1}^{\lambda}=\bigcup_{\mu_{i k_{i}} \in \tilde{h}_{i}, v_{i k_{i}} \in \tilde{g}_{i}} \\
& \quad \times\left\{\left(\left(1-\left(1-\left(\left(1-v_{l+1 k_{l+1}}^{2}\right)^{\lambda}-\left(1-\left(\mu_{l+1 k_{l+1}}^{2}\right.\right.\right.\right.\right.\right.\right. \\
& \left.\left.\left.\left.\left.\quad+v_{l+1 k_{l+1}}^{2}\right)\right)^{\lambda}\right)\right)^{w_{l+1}}\right)^{1 / 2},\left(\left(1-\left(\left(1-v_{l+1 k_{l+1}}^{2}\right)^{\lambda}\right.\right.\right. \\
& \left.\left.-\left(1-\left(\mu_{l+1 k_{l+1}}^{2}+v_{l+1 k_{l+1}}^{2}\right)\right)^{\lambda}\right)\right)^{w_{l+1}} \\
& \left.\left.\left.\quad-\left(1-\left(\mu_{l+1 k_{l+1}}^{2}+v_{l+1 k_{l+1}}^{2}\right)\right)^{w_{l+1} \lambda}\right)^{1 / 2}\right)\right\},
\end{aligned}
$$

$$
\begin{aligned}
& \oplus_{i=1}^{l+1} w_{i} \tilde{f}_{i}^{\lambda}=\left(\oplus_{i=1}^{l} w_{i} \tilde{f}_{i}^{\lambda}\right) \oplus\left(w_{l+1} \alpha_{l+1}^{\lambda}\right) \\
& =\bigcup \\
& \mu_{i k_{i}} \in \tilde{h}_{i}, v_{i k_{i}} \in \tilde{g}_{i} \\
& \times\left\{\left(\left(1-\prod_{i=1}^{l}\left(1-\left(\left(1-v_{i k_{i}}^{2}\right)^{\lambda}\right.\right.\right.\right.\right. \\
& \left.\left.-\left(1-\left(\mu_{i k_{i}}^{2}+v_{i k_{i}}^{2}\right)\right)^{\lambda}\right)\right)^{w_{i}} *\left(\left(1-v_{l+1 k_{l+1}}^{2}\right)^{\lambda}\right. \\
& \left.\left.-\left(1-\left(\mu_{l+1 k_{l+1}}^{2}+v_{l+1 k_{l+1}}^{2}\right)\right)^{\lambda}\right)^{w_{l+1}}\right)^{1 / 2} \text {, } \\
& \times\left(\prod_{i=1}^{l}\left(1-\left(\left(1-v_{i k_{i}}^{2}\right)^{\lambda}-\left(1-\left(\mu_{i k_{i}}^{2}+v_{i k_{i}}^{2}\right)\right)^{\lambda}\right)\right)^{w_{i}}\right. \\
& \times\left(\left(1-v_{l+1 k_{l+1}}^{2}\right)^{\lambda}-\left(1-\left(\mu_{l+1 k_{l+1}}^{2}+v_{l+1 k_{l+1}}^{2}\right)\right)^{\lambda}\right)^{w_{l+1}} \\
& -\prod_{i=1}^{l}\left(1-\left(\mu_{i k_{i}}^{2}+v_{i k_{i}}^{2}\right)\right)^{\lambda w_{i}} \\
& \left.\left.\left.\times\left(1-\left(\mu_{l+1 k_{l+1}}^{2}+v_{l+1 k_{l+1}}^{2}\right)\right)^{\lambda}\right)^{1 / 2}\right)\right\} \\
& =\bigcup \\
& \mu_{i k_{i}} \in \tilde{h}_{i}, v_{i k_{i}} \in \tilde{g}_{i} \\
& \times\left\{\left(\left(1-\prod_{i=1}^{l+1}\left(1-\left(\left(1-v_{i k_{i}}^{2}\right)^{\lambda}\right.\right.\right.\right.\right. \\
& \left.\left.\left.-\left(1-\left(\mu_{i k_{i}}^{2}+v_{i k_{i}}^{2}\right)\right)^{\lambda}\right)\right)^{w_{i}}\right)^{1 / 2} \text {, } \\
& \times\left(\prod_{i=1}^{l+1}\left(1-\left(\left(1-v_{i k_{i}}^{2}\right)^{\lambda}-\left(1-\left(\mu_{i k_{i}}^{2}+v_{i k_{i}}^{2}\right)\right)^{\lambda}\right)^{w_{i}}\right)\right. \\
& \left.\left.\left.-\prod_{i=1}^{l+1}\left(1-\left(\mu_{i k_{i}}^{2}+v_{i k_{i}}^{2}\right)\right)^{\lambda w_{i}}\right)^{1 / 2}\right)\right\} \text {. } \\
& \left(\oplus_{i=1}^{l+1} w_{i} \tilde{f}_{i}^{\lambda}\right)^{1 / \lambda}=\bigcup_{\tilde{n}_{i}, v_{i} \in g^{2}} \\
& \mu_{i k_{i}} \in \tilde{h}_{i}, v_{i k_{i}} \in \tilde{g}_{i} \\
& \times\left\{\left(\left(\left(1-\left(\prod _ { i = 1 } ^ { l + 1 } \left(1-\left(\left(1-v_{i k_{i}}^{2}\right)^{\lambda}\right.\right.\right.\right.\right.\right.\right. \\
& \left.\left.\left.-\left(1-\left(\mu_{i k_{i}}^{2}+v_{i k_{i}}^{2}\right)\right)^{\lambda}\right)\right)^{w_{i}}\right) \\
& \left.-\prod_{i=1}^{l+1}\left(1-\left(\mu_{i k_{i}}^{2}+v_{i k_{i}}^{2}\right)\right)^{\lambda w_{i}}\right)^{1 / \lambda} \\
& \left.-\prod_{i=1}^{k+1}\left(1-\left(\mu_{i k_{i}}^{2}+v_{i k_{i}}^{2}\right)\right)^{w_{i}}\right)^{1 / 2} \text {, } \\
& \times\left(1-\left(1-\left(\prod _ { i = 1 } ^ { l + 1 } \left(1-\left(\left(1-v_{i k_{i}}^{2}\right)^{\lambda}\right.\right.\right.\right.\right. \\
& \left.\left.-\left(1-\left(\mu_{i k_{i}}^{2}+v_{i k_{i}}^{2}\right)\right)^{\lambda}\right)^{w_{i}}\right) \\
& \left.\left.\left.\left.\left.-\prod_{i=1}^{l+1}\left(1-\left(\mu_{i k_{i}}^{2}+v_{i k_{i}}^{2}\right)\right)^{\lambda w_{i}}\right)\right)^{1 / \lambda}\right)^{1 / 2}\right)\right\} \text {. }
\end{aligned}
$$

Then Eq. (12) holds for $n=l+1$. Hence, Eq. (12) holds for all $n$ from mathematical induction. Moreover, for each $(\mu, v)$ in the GHPFIWA operator, 
$\left.\left.\mu^{2}+v^{2}=1-\prod_{i=1}^{k+1}\left(1-\left(\mu_{i k_{i}}^{2}+v_{i k_{i}}^{2}\right)\right)^{\lambda w_{i}}\right)\right)^{1 / \lambda}$,

Since $0 \leq \mu_{i k_{i}}^{2}+v_{i k_{i}}^{2} \leq 1$, then $0 \leq \mu^{2}+v^{2} \leq 1$. The aggregated result of the GHPFIWA operator is still an HPFE.

Theorem 9 Let $\tilde{f}_{i}=\left(\tilde{h}_{i}, \tilde{g}_{i}\right)(i=1,2, \ldots, n)$ be a collection of HPFEs. If all the HPFEs reduce to $\tilde{f}=(\tilde{h}, \tilde{g})$, the GHPFIWA operator reduces to the following form

$\operatorname{GHPFIWA}\left(\tilde{f}_{1}, \tilde{f}_{2}, \ldots, \tilde{f}_{n}\right)=\tilde{f}$

Theorem 10 Let $\tilde{f}_{i}=\left(\tilde{h}_{i}, \tilde{g}_{i}\right)(i=1,2, \ldots, n)$ be a collection of HPFEs. Let $\tilde{f}^{+}=(1,0), \tilde{f}^{-}=(0,1)$, then

$\tilde{f}^{-} \leq \operatorname{GHPFIWA}\left(\tilde{f}_{1}, \tilde{f}_{2}, \ldots, \tilde{f}_{n}\right) \leq \tilde{f}^{+}$.

If the weight vector is taken as $\left(\frac{1}{n}, \frac{1}{n}, \ldots, \frac{1}{n}\right)$, the GHPFIWA $\lambda$ operator reduces to the generalized Pythagorean fuzzy interaction averaging (GHPFIA) operator as follows

$$
\begin{aligned}
& \operatorname{GHPFIA}\left(\tilde{f}_{1}, \tilde{f}_{2}, \ldots, \tilde{f}_{n}\right)=\left(\oplus_{j=1}^{n} \frac{1}{n} \tilde{f}_{j}^{\lambda}\right)^{1 / \lambda} \\
& =\bigcup \\
& \mu_{i k_{i}} \in \tilde{h}_{i}, v_{i k_{i}} \in \tilde{g}_{i} \\
& \times\left\{\left(\left(( 1 - ) \prod _ { i = 1 } ^ { k + 1 } \left(1-\left(\left(1-v_{i k_{i}}^{2}\right)^{\lambda}\right.\right.\right.\right.\right. \\
& \left.\left.\left.-\left(1-\left(\mu_{i k_{i}}^{2}+v_{i k_{i}}^{2}\right)\right)^{\lambda}\right)\right)^{\frac{1}{n}}\right) \\
& \left.-\prod_{i=1}^{k+1}\left(1-\left(\mu_{i k_{i}}^{2}+v_{i k_{i}}^{2}\right)\right)^{\frac{\lambda}{n}}\right)^{1 / \lambda} \\
& \left.-\prod_{i=1}^{k+1}\left(1-\left(\mu_{i k_{i}}^{2}+v_{i k_{i}}^{2}\right)\right)^{\frac{1}{n}}\right)^{1 / 2} \text {, } \\
& \times\left(1-\left(1-\left(\prod _ { i = 1 } ^ { k + 1 } \left(1-\left(\left(1-v_{i k_{i}}^{2}\right)^{\lambda}\right.\right.\right.\right.\right. \\
& \left.\left.-\left(1-\left(\mu_{i k_{i}}^{2}+v_{i k_{i}}^{2}\right)\right)^{\lambda}\right)^{\frac{1}{n}}\right) \\
& \left.\left.\left.\left.\left.-\prod_{i=1}^{k+1}\left(1-\left(\mu_{i k_{i}}^{2}+v_{i k_{i}}^{2}\right)\right)^{\frac{\lambda}{n}}\right)\right)^{1 / \lambda}\right)^{1 / 2}\right)\right\} \text {. }
\end{aligned}
$$

Example 3 Suppose the Pythagorean fuzzy values are the same as that in Example 1. Using the GHPFIWA operator, we can get $\operatorname{GHPFIWA}\left(\tilde{f}_{1}, \tilde{f}_{2}, \tilde{f}_{3}\right)=\{(0.5590,0.1800)$, $(0.4870,0.2125)\}$.

\section{Hesitant Pythagorean fuzzy interaction Bonferroni mean operators}

The Bonferroni mean aggregation operator was defined by Bonferroni [51] in 1950. It was generalized by Yager [52] and others.
Definition 13 Let $\tilde{f}_{i}=\left(\tilde{h}_{i}, \tilde{g}_{i}\right)(i=1,2, \ldots, n)$ be a collection of HPFEs. For any $p, q \geq 0$ with $p+q>0$, the hesitant Pythagorean fuzzy interaction Bonferroni mean (HPFIBM) aggregation operator can be defined as

$\operatorname{HPFIBM}^{p, q}\left(\tilde{f}_{1}, \tilde{f}_{2}, \ldots, \tilde{f}_{n}\right)$

$$
=\left(\frac{1}{n(n-1)} \oplus_{i, j=1, i \neq j}^{n}\left(\tilde{f}_{i}^{p} \otimes \tilde{f}_{j}^{q}\right)\right)^{\frac{1}{p+q}} .
$$

Theorem 11 Let $\tilde{f}_{i}=\left(\tilde{h}_{i}, \tilde{g}_{i}\right)(i=1,2, \ldots, n)$ be a collection of HPFEs. Then, the aggregated result of the HPFIBM operator is still an HPFE, which has the following form

$$
\begin{aligned}
& \operatorname{HPFIBM}^{p, q}\left(\tilde{f}_{1}, \tilde{f}_{2}, \ldots, \tilde{f}_{n}\right) \\
& =\left(\frac{1}{n(n-1)} \oplus_{i, j=1, i \neq j}^{n}\left(\tilde{f}_{i}^{p} \otimes \tilde{f}_{j}^{q}\right)\right)^{\frac{1}{p+q}} \\
& =\bigcup \\
& \mu_{i k_{i}} \in \tilde{h}_{i}, v_{i k_{i}} \in \tilde{g}_{i}, \mu_{j k_{j}} \in \tilde{h}_{j}, v_{j k_{j}} \in \tilde{g}_{j} \\
& \times\left\{\left(\left(\left(1-\prod_{i, j=1, i \neq j}^{n}\left(1-\left(1-v_{i k_{i}}^{2}\right)^{p}\left(1-v_{j k_{j}}^{2}\right)^{q}\right.\right.\right.\right.\right. \\
& \left.-\left(1-\left(\mu_{i k_{i}}^{2}+v_{i k_{i}}^{2}\right)\right)^{p}\left(1-\left(\mu_{j k_{j}}^{2}+v_{j k_{j}}^{2}\right)\right)^{q}\right)^{\frac{1}{n(n-1)}} \\
& +\prod_{i, j=1, i \neq j}^{n}\left(\left(1-\left(\mu_{i k_{i}}^{2}+v_{i k_{i}}^{2}\right)\right)^{p}\right. \\
& \left.\left.\times\left(1-\left(\mu_{j k_{j}}^{2}+v_{j k_{j}}^{2}\right)\right)^{q}\right)^{\frac{1}{n(n-1)}}\right)^{\frac{1}{p+q}} \\
& -\prod_{i, j=1, i \neq j}^{n}\left(\left(1-\left(\mu_{i k_{i}}^{2}+v_{i k_{i}}^{2}\right)\right)^{p} *\right. \\
& \left.\left.\times\left(1-\left(\mu_{j k_{j}}^{2}+v_{j k_{j}}^{2}\right)\right)^{q}\right)^{\frac{1}{n(n-1)(p+q)}}\right)^{1 / 2} \text {, } \\
& \times\left(1-\left(1-\prod_{i, j=1, i \neq j}^{n}\left(1-\left(1-v_{i k_{i}}^{2}\right)^{p} *\right.\right.\right. \\
& \times\left(1-v_{j k_{j}}^{2}\right)^{q}-\left(1-\left(\mu_{i k_{i}}^{2}+v_{i k_{i}}^{2}\right)\right)^{p} \\
& \left.\left(1-\left(\mu_{j k_{j}}^{2}+v_{j k_{j}}^{2}\right)\right)^{q}\right)^{\frac{1}{n(n-1)}} \\
& +\prod_{i, j=1, i \neq j}^{n}\left(\left(1-\left(\mu_{i k_{i}}^{2}+v_{i k_{i}}^{2}\right)\right)^{p}\right. \\
& \left.\left.\left.\left.\left.\left(1-\left(\mu_{j k_{j}}^{2}+v_{j k_{j}}^{2}\right)\right)^{q}\right)^{\frac{1}{n(n-1)}}\right)^{\frac{1}{p+q}}\right)^{1 / 2}\right)\right\} \text {. }
\end{aligned}
$$

Proof Since

$$
\begin{aligned}
& \tilde{f}_{i}^{p}=\bigcup_{\mu_{i k_{i}} \in \tilde{h}_{i}, v_{i k_{i}} \in \tilde{g}_{i}} \\
& \times\left\{\left(\sqrt{\left(1-v_{i k_{i}}^{2}\right)^{p}-\left(1-\left(\mu_{i k_{i}}^{2}+v_{i k_{i}}^{2}\right)\right)^{p}},\right.\right. \\
& \left.\left.\times \sqrt{1-\left(1-v_{i k_{i}}^{2}\right)^{p}}\right)\right\} \\
& \tilde{f}_{j}^{q}=\bigcup_{\mu_{j k_{j}} \in \tilde{g}_{j}, v_{j k_{j}} \in \tilde{h}_{j}}
\end{aligned}
$$




$$
\begin{aligned}
& \times\left\{\left(\sqrt{\left(1-v_{j k_{j}}^{2}\right)^{q}-\left(1-\left(\mu_{j k_{j}}^{2}+v_{j k_{j}}^{2}\right)^{q}\right.},\right.\right. \\
& \left.\left.\sqrt{1-\left(1-v_{j k_{j}}^{2}\right)^{q}}\right)\right\} \text {, } \\
& \tilde{f}_{i}^{p} \otimes \tilde{f}_{j}^{q}= \\
& \mu_{i k_{i}} \in \tilde{h}_{i}, v_{i k_{i}} \in \tilde{g}_{i}, \mu_{j k_{j}} \in \tilde{h}_{j}, v_{j k_{j}} \in \tilde{g}_{j} \\
& \times\left\{\left(\left(\left(1-v_{i k_{i}}^{2}\right)^{p}\left(1-v_{j k_{j}}^{2}\right)^{q}\right.\right.\right. \\
& \left.-\left(1-\left(\mu_{i k_{i}}^{2}+v_{i k_{i}}^{2}\right)\right)^{p}\left(1-\left(\mu_{j k_{j}}^{2}+v_{j k_{j}}^{2}\right)\right)^{q}\right)^{1 / 2}, \\
& \left.\left.\times \sqrt{1-\left(1-v_{i k_{i}}^{2}\right)^{p}\left(1-v_{j k_{j}}^{2}\right)^{q}}\right)\right\}, \\
& \oplus_{i, j=1, i \neq j}^{n}\left(\tilde{f}_{i}^{p} \otimes \tilde{f}_{j}^{q}\right) \\
& =\bigcup_{\mu_{i k_{i}} \in \tilde{h}_{i}, v_{i k_{i}} \in \tilde{g}_{i}, \mu_{j k_{j}} \in \tilde{h}_{j}, v_{j k_{j}} \in \tilde{g}_{j}} \\
& \times\left\{\left(\left(1-\prod_{i, j=1, i \neq j}^{n}\left(1-\left(1-v_{i k_{i}}^{2}\right)^{p}\left(1-v_{j k_{j}}^{2}\right)^{q}\right.\right.\right.\right. \\
& \left.\left.+\left(1-\left(\mu_{i k_{i}}^{2}+v_{i k_{i}}^{2}\right)\right)^{p}\left(1-\left(\mu_{j k_{j}}^{2}+v_{j k_{j}}^{2}\right)\right)^{q}\right)\right)^{1 / 2}, \\
& \times\left(\prod _ { i , j = 1 , i \neq j } ^ { n } \left(1-\left(1-v_{i k_{i}}^{2}\right)^{p}(1\right.\right. \\
& \left.\left.-v_{j k_{j}}^{2}\right)^{q}-\left(1-\left(\mu_{i k_{i}}^{2}+v_{i k_{i}}^{2}\right)\right)^{p}\left(1-\left(\mu_{j k_{j}}^{2}+v_{j k_{j}}^{2}\right)\right)^{q}\right) \\
& -\prod_{i, j=1, i \neq j}^{n}\left(1-\left(\mu_{i k_{i}}^{2}\right.\right. \\
& \left.\left.\left.\left.\left.+v_{i k_{i}}^{2}\right)\right)^{p}\left(1-\left(\mu_{j k_{j}}^{2}+v_{j k_{j}}^{2}\right)\right)^{q}\right)^{1 / 2}\right)\right\} \text {, } \\
& \frac{1}{n(n-1)} \oplus_{i, j=1, i \neq j}^{n}\left(\tilde{f}_{i}^{p} \otimes \tilde{f}_{j}^{q}\right)
\end{aligned}
$$

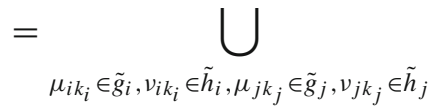

$$
\begin{aligned}
& \times\left\{\left(\left(1-\prod_{i, j=1, i \neq j}^{n}\left(1-\left(1-v_{i k_{i}}^{2}\right)^{p}\left(1-v_{j k_{j}}^{2}\right)^{q}\right.\right.\right.\right. \\
& \left.\left.+\left(1-\left(\mu_{i k_{i}}^{2}+v_{i k_{i}}^{2}\right)\right)^{p}\left(1-\left(\mu_{j k_{j}}^{2}+v_{j k_{j}}^{2}\right)\right)^{q}\right)^{\frac{1}{n(n-1)}}\right)^{1 / 2}, \\
& \times\left(\prod_{i, j=1, i \neq j}^{n}(1-(1\right. \\
& \left.-v_{i k_{i}}^{2}\right)^{p}\left(1-v_{j k_{j}}^{2}\right)^{q} \\
& \left.-\left(1-\left(\mu_{i k_{i}}^{2}+v_{i k_{i}}^{2}\right)\right)^{p}\left(1-\left(\mu_{j k_{j}}^{2}+v_{j k_{j}}^{2}\right)\right)^{q}\right)^{\frac{1}{n(n-1)}} \\
& -\prod_{i, j=1, i \neq j}^{n}\left(\left(1-\left(\mu_{i k_{i}}^{2}+v_{i k_{i}}^{2}\right)\right)^{p}\right. \\
& \left.\left.\left.\left.\times\left(1-\left(\mu_{j k_{j}}^{2}+v_{j k_{j}}^{2}\right)\right)^{q}\right)^{\frac{1}{n(n-1)}}\right)^{1 / 2}\right)\right\} \text {, } \\
& \times\left(\frac{1}{n(n-1)} \oplus_{i, j=1, i \neq j}^{n}\left(\tilde{f}_{i}^{p} \otimes \tilde{f}_{j}^{q}\right)\right)^{\frac{1}{p+q}} \\
& =\bigcup \\
& \mu_{i k_{i}} \in \tilde{g}_{i}, v_{i k_{i}} \in \tilde{h}_{i}, \mu_{j k_{j}} \in \tilde{g}_{j}, v_{j k_{j}} \in \tilde{h}_{j} \\
& \times\left\{\left(\left(\left(1-\prod_{i, j=1, i \neq j}^{n}\left(1-\left(1-v_{i k_{i}}^{2}\right)^{p}\left(1-v_{j k_{j}}^{2}\right)^{q}-\right.\right.\right.\right.\right.
\end{aligned}
$$

$$
\begin{aligned}
& \left.\times\left(1-\left(\mu_{i k_{i}}^{2}+v_{i k_{i}}^{2}\right)\right)^{p}\left(1-\left(\mu_{j k_{j}}^{2}+v_{j k_{j}}^{2}\right)\right)^{q}\right)^{\frac{1}{n(n-1)}} \\
& +\prod_{i, j=1, i \neq j}^{n}\left(\left(1-\left(\mu_{i k_{i}}^{2}\right.\right.\right. \\
& \left.\left.\left.\left.+v_{i k_{i}}^{2}\right)\right)^{p}\left(1-\left(\mu_{j k_{j}}^{2}+v_{j k_{j}}^{2}\right)\right)^{q}\right)^{\frac{1}{n(n-1)}}\right)^{\frac{1}{p+q}} \\
& -\prod_{i, j=1, i \neq j}^{n}\left(\left(1-\left(\mu_{i k_{i}}^{2}+v_{i k_{i}}^{2}\right)\right)^{p} *\right. \\
& \left.\left.\times\left(1-\left(\mu_{j k_{j}}^{2}+v_{j k_{j}}^{2}\right)\right)^{q}\right)^{\frac{1}{n(n-1)(p+q)}}\right)^{1 / 2}, \\
& \times\left(1-\left(1-\prod_{i, j=1, i \neq j}^{n}\left(1-\left(1-v_{i k_{i}}^{2}\right)^{p} *\right.\right.\right. \\
& \times\left(1-v_{j k_{j}}^{2}\right)^{q}-\left(1-\left(\mu_{i k_{i}}^{2}+v_{i k_{i}}^{2}\right)\right)^{p}(1- \\
& \left.\left.\times\left(\mu_{j k_{j}}^{2}+v_{j k_{j}}^{2}\right)\right)^{q}\right)^{\frac{1}{n(n-1)}} \\
& +\prod_{i, j=1, i \neq j}^{n}\left(( 1 - ( \mu _ { i k _ { i } } ^ { 2 } q + v _ { i k _ { i } } ^ { 2 } ) ) ^ { p } \left(1-\left(\mu_{j k_{j}}^{2}\right.\right.\right. \\
& \left.\left.\left.\left.\left.\left.\left.+v_{j k_{j}}^{2}\right)\right)^{q}\right)^{\frac{1}{n(n-1)}}\right)^{\frac{1}{p+q}}\right)^{1 / 2}\right)\right\} .
\end{aligned}
$$

Moreover, for each $(\mu, v)$ in the aggregated result of the HPFIBM operator, we have

$$
\begin{aligned}
\mu^{2}+v^{2} & =1-\prod_{i, j=1, i \neq j}^{n}\left(\left(1-\left(\mu_{i k_{i}}^{2}\right.\right.\right. \\
& \left.\left.\left.+v_{i k_{i}}^{2}\right)\right)^{p}\left(1-\left(\mu_{j k_{j}}^{2}+v_{j k_{j}}^{2}\right)\right)^{q}\right)^{\frac{1}{n(n-1)(p+q)}} .
\end{aligned}
$$

Since $0 \leq \mu_{i k_{i}}^{2}+v_{i k_{i}}^{2} \leq 1$ and $0 \leq \mu_{j k_{j}}^{2}+v_{j k_{j}}^{2} \leq 1$, hence $0 \leq \mu^{2}+v^{2} \leq 1$. Then, the aggregated result of the HPFIBM operator is still an HPFE.

Theorem 12 Let $\tilde{f}_{i}=\left(\tilde{h}_{i}, \tilde{g}_{i}\right)(i=1,2, \ldots, n)$ be a collection of HPFEs. If all the HPFEs reduce to $\tilde{f}=(\tilde{h}, \tilde{g})$, the HPFIBM operator reduces to the following form

$\operatorname{HPFIBM}\left(\tilde{f}_{1}, \tilde{f}_{2}, \ldots, \tilde{f}_{n}\right)=\tilde{f}$.

Theorem 13 Let $\tilde{f}_{i}=\left(\tilde{h}_{i}, \tilde{g}_{i}\right)(i=1,2, \ldots, n)$ be a collection of HPFEs. Let $\tilde{f}^{+}=(1,0), \tilde{f}^{-}=(0,1)$, then

$\tilde{f}^{-} \leq \operatorname{HPFIBM}\left(\tilde{f}_{1}, \tilde{f}_{2}, \ldots, \tilde{f}_{n}\right) \leq \tilde{f}^{+}$

Example 4 Suppose the Pythagorean fuzzy values are the same as that in Example 1. Using the HPFIBM operator, we can get $\operatorname{HPFIBM}\left(\tilde{f}_{1}, \tilde{f}_{2}, \tilde{f}_{3}\right)=\{(0.7931,0.3155),(0.7823$, $0.3234),(0.7701,0.3515),(0.7576,0.3607)\}$.

Definition 14 Let $\tilde{f}_{i}=\left(\tilde{h}_{i}, \tilde{g}_{i}\right)(i=1,2, \ldots, n)$ be a collection of HPFEs. For any $p, q \geq 0$ with $p+q>0$, the hesitant Pythagorean fuzzy interaction weighted Bonferroni mean (HPFIWBM) aggregation operator can be defined as 
$\operatorname{HPFIWBM}^{p, q}\left(\tilde{f}_{1}, \tilde{f}_{2}, \ldots, \tilde{f}_{n}\right)$

$$
=\left(\frac{1}{n(n-1)} \oplus_{i, j=1, i \neq j}^{n}\left(\left(w_{i} \tilde{f}_{i}\right)^{p} \otimes\left(w_{j} \tilde{f}_{j}\right)^{q}\right)\right)^{\frac{1}{p+q}},
$$

where $\left(w_{1}, w_{2}, \ldots, w_{n}\right)$ is the weight vector satisfying $w_{i} \geq$ $0, \sum_{i=1}^{n} w_{i}=1$.

Theorem 14 Let $\tilde{f}_{i}=\left(\tilde{h}_{i}, \tilde{g}_{i}\right)(i=1,2, \ldots, n)$ be a collection of HPFEs. Then, the aggregated result of the HPFIWBM operator is still an HPFE, which has the following form

$$
\begin{array}{rl}
H & F I W B M^{p, q}\left(\tilde{f}_{1}, \tilde{f}_{2}, \ldots, \tilde{f}_{n}\right) \\
= & \left(\frac{1}{n(n-1)} \oplus_{i, j=1, i \neq j}^{n}\left(\left(w_{i} \tilde{f}_{i}\right)^{p} \otimes\left(w_{j} \tilde{f}_{j}\right)^{q}\right)\right)^{\frac{1}{p+q}} \\
= & \bigcup \\
& \mu_{i k_{i} \in \tilde{h}_{i}, v_{i k_{i}} \in \tilde{g}_{i}, \mu_{j k_{j}} \in \tilde{h}_{j}, v_{j k_{j}} \in \tilde{g}_{j}} \\
& \times\left\{\left(\left(\left(1-\prod_{i, j=1, i \neq j}^{n}\left(1-\left(1-\left(1-\mu_{i k_{i}}^{2}\right)^{w_{i}}\right.\right.\right.\right.\right.\right. \\
& \left.+\left(1-\left(\mu_{i k_{i}}^{2}+v_{i k_{i}}^{2}\right)\right)^{w_{i}}\right)^{p}\left(1-\left(1-\mu_{j k_{j}}^{2}\right)^{q}\right. \\
& \left.+\left(1-\left(\mu_{j k_{j}}^{2}+v_{j k_{j}}^{2}\right)\right)^{w_{j}}\right)^{q}+(1 \\
& \left.\left.-\left(\mu_{i k_{i}}^{2}+v_{i k_{i}}^{2}\right)\right)^{w_{i} p}\left(1-\left(\mu_{j k_{j}}^{2}+v_{j k_{j}}^{2}\right)\right)^{w_{j} q}\right)^{\frac{1}{n(n-1)}} \\
& +\prod_{i, j=1, i \neq j}^{n}\left(\left(1-\left(\mu_{i k_{i}}^{2}\right.\right.\right. \\
& \left.\left.\left.\left.+v_{i k_{i}}^{2}\right)\right)^{w_{i} p}\left(1-\left(\mu_{j k_{j}}^{2}+v_{j k_{j}}^{2}\right)\right)^{w_{j} q}\right)^{\frac{1}{n(n-1)}}\right)^{\frac{1}{p+q}} \\
& -\prod_{i, j=1, i \neq j}^{n}\left(\left(1-\left(\mu_{i k_{i}}^{2}\right.\right.\right. \\
& \left.\left.\left.\left.+v_{i k_{i}}^{2}\right)\right)^{w_{i} p}\left(1-\left(\mu_{j k_{j}}^{2}+v_{j k_{j}}^{2}\right)\right)^{w_{j} q}\right)^{\frac{1}{n(n-1)(p+q)}}\right)^{1 / 2}, \\
& \times\left(1-\left(1-\prod_{i, j=1, i \neq j}^{n}(1\right.\right. \\
& -\left(1-\left(1-\mu_{i k_{i}}^{2}\right)^{w_{i}}+\left(1-\left(\mu_{i k_{i}}^{2}+v_{i k_{i}}^{2}\right)\right)^{w_{i}}\right)^{p}(1 \\
& -\left(1-\mu_{j k_{j}}^{2}\right)^{q}+\left(1-\left(\mu_{j k_{j}}^{2}\right.\right. \\
& \left.\left.\left.+v_{j k_{j}}^{2}\right)\right)^{w_{j}}\right)^{q}+\left(1-\left(\mu_{i k_{i}}^{2}+v_{i k_{i}}^{2}\right)\right)^{w_{i} p}(1 \\
& \left.\left.-\left(\mu_{j k_{j}}^{2}+v_{j k_{j}}^{2}\right)\right)^{w_{j} q}\right)^{\frac{1}{n(n-1)}} \\
& \times \prod_{i, j=1, i \neq j}^{n}\left(\left(1-\left(\mu_{i k_{i}}^{2}\right.\right.\right. \\
& \left.\left.\left.\left.\left.\left.\left.+v_{i k_{i}}^{2}\right)\right)^{w_{i} p}\left(1-\left(\mu_{j k_{j}}^{2}+v_{j k_{j}}^{2}\right)\right)^{w_{j} q}\right)^{\frac{1}{n(n-1)}}\right)^{\frac{1}{p+q}}\right)^{1 / 2}\right)\right\} .
\end{array}
$$

Definition 15 Let $\tilde{f}_{i}=\left(\tilde{h}_{i}, \tilde{g}_{i}\right)(i=1,2, \ldots, n)$ be a collection of HPFEs. For any $p, q \geq 0$ with $p+q>0$, the hesitant Pythagorean fuzzy interaction geometric Bonferroni mean (HPFIGBM) aggregation operator can be defined as
$\operatorname{HPFIGBM}^{p, q}\left(\tilde{f}_{1}, \tilde{f}_{2}, \ldots, \tilde{f}_{n}\right)$

$$
=\frac{1}{p+q} \otimes_{i, j=1, i \neq j}^{n}\left(p \tilde{f}_{i} \oplus q \tilde{f}_{j}\right)^{\frac{1}{n(n-1)}}
$$

Theorem 15 Let $\tilde{f}_{i}=\left(\tilde{h}_{i}, \tilde{g}_{i}\right)(i=1,2, \ldots, n)$ be a collection of HPFEs. Then

$$
\begin{aligned}
& \operatorname{HPFIGBM}^{p, q}\left(\tilde{f}_{1}, \tilde{f}_{2}, \ldots, \tilde{f}_{n}\right) \\
& =\frac{1}{p+q} \otimes_{i, j=1, i \neq j}^{n}\left(p \tilde{f}_{i} \oplus q \tilde{f}_{j}\right)^{\frac{1}{n(n-1)}} \\
& =\quad \bigcup \\
& \mu_{i k_{i}} \in \tilde{h}_{i}, v_{i k_{i}} \in \tilde{g}_{i}, \mu_{j k_{j}} \in \tilde{h}_{j}, v_{j k_{j}} \in \tilde{g}_{j} \\
& \times\left\{\left(\left(1-\left(1-\prod_{i, j=1, i \neq j}^{n}\left(1-\left(1-\mu_{i k_{i}}^{2}\right)^{p}(1\right.\right.\right.\right.\right. \\
& \left.-\mu_{j k_{j}}^{2}\right)^{q}+\left(1-\left(\mu_{i k_{i}}^{2}+v_{i k_{i}}^{2}\right)\right)^{p}(1 \\
& \left.\left.-\left(\mu_{j k_{j}}^{2}+v_{j k_{j}}^{2}\right)\right)^{q}\right)^{\frac{1}{n(n-1)}}+\prod_{i, j=1, i \neq j}^{n}((1 \\
& \left.-\left(\mu_{i k_{i}}^{2}+v_{i k_{i}}^{2}\right)\right)^{p}\left(1-\left(\mu_{j k_{j}}^{2}\right.\right. \\
& \left.\left.\left.\left.\left.+v_{j k_{j}}^{2}\right)\right)^{q}\right)^{\frac{1}{n(n-1)}}\right)^{\frac{1}{p+q}}\right)^{1 / 2} \\
& \times\left(\left(1-\prod_{i, j=1, i \neq j}^{n}(1\right.\right. \\
& -\left(1-\mu_{i k_{i}}^{2}\right)^{p}\left(1-\mu_{j k_{j}}^{2}\right)^{q}+\left(1-\left(\mu_{i k_{i}}^{2}+v_{i k_{i}}^{2}\right)\right)^{p}(1 \\
& \left.\left.-\left(\mu_{j k_{j}}^{2}+v_{j k_{j}}^{2}\right)\right)^{q}\right)^{\frac{1}{n(n-1)}} \\
& +\prod_{i, j=1, i \neq j}^{n}\left(\left(1-\left(\mu_{i k_{i}}^{2}+v_{i k_{i}}^{2}\right)\right)^{p}(1\right. \\
& \left.\left.\left.-\left(\mu_{j k_{j}}^{2}+v_{j k_{j}}^{2}\right)\right)^{q}\right)^{\frac{1}{n(n-1)}}\right)^{\frac{1}{p+q}} \\
& -\prod_{i, j=1, i \neq j}^{n}\left(\left(1-\left(\mu_{i k_{i}}^{2}+v_{i k_{i}}^{2}\right)\right)^{p}(1\right. \\
& \left.\left.\left.\left.\left.-\left(\mu_{j k_{j}}^{2}+v_{j k_{j}}^{2}\right)\right)^{q}\right)^{\frac{1}{n(n-1)(p+q)}}\right)^{1 / 2}\right)\right\} \text {. }
\end{aligned}
$$

Proof

$$
\begin{aligned}
& p \tilde{f_{i}}=\bigcup \bigcup \\
& \mu_{i k_{i}} \in \tilde{h}_{i}, v_{i k_{i}} \in \tilde{g}_{i} \\
& \times\left\{\left(\sqrt{1-\left(1-\mu_{i k_{i}}^{2}\right)^{p}},\left(\left(1-\mu_{i k_{i}}^{2}\right)^{p}\right.\right.\right. \\
& \left.\left.\left.-\left(1-\left(\mu_{i k_{i}}^{2}+v_{i k_{i}}^{2}\right)\right)^{p}\right)^{1 / 2}\right)\right\}, \\
& q \tilde{f}_{j}=\bigcup_{\mu_{j k_{j}} \in \tilde{h}_{j}, v_{j k_{j}} \in \tilde{g}_{j}} \\
& \times\left\{\left(\sqrt{1-\left(1-\mu_{j k_{j}}^{2}\right)^{q}},\left(\left(1-\mu_{j k_{j}}^{2}\right)^{q}\right.\right.\right. \\
& \left.\left.\left.-\left(1-\left(\mu_{j k_{j}}^{2}+v_{j k_{j}}^{2}\right)\right)^{q}\right)^{1 / 2}\right)\right\}, \\
& p \tilde{f}_{i} \oplus q \tilde{f}_{j}
\end{aligned}
$$




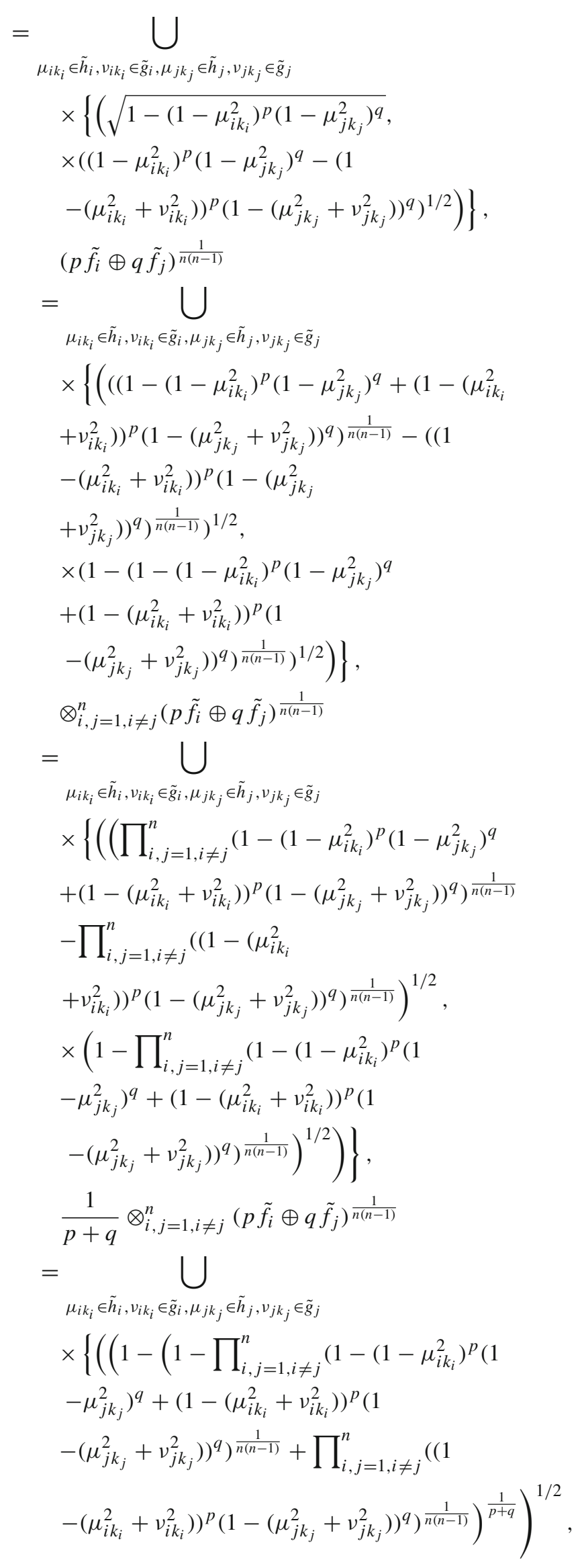

$$
\begin{aligned}
& \times\left(\left(1-\prod_{i, j=1, i \neq j}^{n}(1\right.\right. \\
& -\left(1-\mu_{i k_{i}}^{2}\right)^{p}\left(1-\mu_{j k_{j}}^{2}\right)^{q}+\left(1-\left(\mu_{i k_{i}}^{2}+v_{i k_{i}}^{2}\right)\right)^{p}(1 \\
& \left.\left.-\left(\mu_{j k_{j}}^{2}+v_{j k_{j}}^{2}\right)\right)^{q}\right)^{\frac{1}{n(n-1)}} \\
& +\prod_{i, j=1, i \neq j}^{n}\left(( 1 - ( \mu _ { i k _ { i } } ^ { 2 } + v _ { i k _ { i } } ^ { 2 } ) ) ^ { p } \left(1-\left(\mu_{j k_{j}}^{2}\right.\right.\right. \\
& \left.\left.\left.\left.+v_{j k_{j}}^{2}\right)\right)^{q}\right)^{\frac{1}{n(n-1)}}\right)^{\frac{1}{p+q}} \\
& -\prod_{i, j=1, i \neq j}^{n}\left(( 1 - ( \mu _ { i k _ { i } } ^ { 2 } + v _ { i k _ { i } } ^ { 2 } ) ) ^ { p } \left(1-\left(\mu_{j k_{j}}^{2}\right.\right.\right. \\
& \left.\left.\left.\left.\left.\left.+v_{j k_{j}}^{2}\right)\right)^{q}\right)^{\frac{1}{n(n-1)(p+q)}}\right)^{1 / 2}\right)\right\} .
\end{aligned}
$$

Moreover, for each $(\mu, v)$ in the HPFIGBM operator,

$$
\begin{aligned}
& \mu^{2}+v^{2}=1-\prod_{i, j=1, i \neq j}^{n}\left(\left(1-\left(\mu_{i k_{i}}^{2}\right.\right.\right. \\
& \left.\left.\left.+v_{i k_{i}}^{2}\right)\right)^{p}\left(1-\left(\mu_{j k_{j}}^{2}+v_{j k_{j}}^{2}\right)\right)^{q}\right)^{\frac{1}{n(n-1)(p+q)}} .
\end{aligned}
$$

Since $0 \leq \mu_{i k_{i}}^{2}+v_{i k_{i}}^{2} \leq 1$ and $0 \leq \mu_{j k_{j}}^{2}+v_{j k_{j}}^{2} \leq 1$, then $0 \leq \mu^{2}+v^{2} \leq 1$. Hence, the aggregated result of the HPFIGBM operator is still an HPFE.

Theorem 16 Let $\tilde{f}_{i}=\left(\tilde{h}_{i}, \tilde{g}_{i}\right)(i=1,2, \ldots, n)$ be a collection of HPFEs. If all the HPFEs reduce to $\tilde{f}=(\tilde{h}, \tilde{g})$, the HPFIGBM operator reduces to the following form

$\operatorname{HPFIGBM}\left(\tilde{f}_{1}, \tilde{f}_{2}, \ldots, \tilde{f}_{n}\right)=\tilde{f}$.

Theorem 17 Let $\tilde{f}_{i}=\left(\tilde{h}_{i}, \tilde{g}_{i}\right)(i=1,2, \ldots, n)$ be a collection of HPFEs. Let $\tilde{f}^{+}=(1,0), \tilde{f}^{-}=(0,1)$, then

$\tilde{f}^{-} \leq \operatorname{HPFIGBM}\left(\tilde{f}_{1}, \tilde{f}_{2}, \ldots, \tilde{f}_{n}\right) \leq \tilde{f}^{+}$.

Example 5 Suppose the Pythagorean fuzzy values are the same as that in Example 1. Using the HPFIGBM operator, we can get $\operatorname{HPFIGBM}\left(\tilde{f}_{1}, \tilde{f}_{2}, \tilde{f}_{3}\right)=\{(0.7804,0.3457)$, (0.7429, 0.4058), (0.7752, 0.3399), (0.7389, 0.3975)\}.

Definition 16 Let $\tilde{f}_{i}=\left(\tilde{h}_{i}, \tilde{g}_{i}\right)(i=1,2, \ldots, n)$ be a collection of HPFEs. For any $p, q \geq 0$ with $p+q>0$, the hesitant Pythagorean fuzzy interaction geometric weight Bonferroni mean (HPFIGWBM) aggregation operator can be defined as

$\operatorname{HPFIGWBM}^{p, q}\left(\tilde{f}_{1}, \tilde{f}_{2}, \ldots, \tilde{f}_{n}\right)$

$$
=\frac{1}{p+q} \otimes_{i, j=1, i \neq j}^{n}\left(p \tilde{f}_{i}^{w_{i}} \oplus q \tilde{f}_{j}^{w_{j}}\right)^{\frac{1}{n(n-1)}},
$$

where $\left(w_{1}, w_{2}, \ldots, w_{n}\right)$ is the weight vector satisfying $w_{i} \geq$ $0, \sum_{i=1}^{n} w_{i}=1$. 
Theorem 18 Let $\tilde{f}_{i}=\left(\tilde{h}_{i}, \tilde{g}_{i}\right)(i=1,2, \ldots, n)$ be a collection of HPFEs. $\left(w_{1}, w_{2}, \ldots, w_{n}\right)$ is the weight vector with $w_{i} \geq 0, \sum_{i=1}^{n} w_{i}=1$. Then

$$
\begin{aligned}
& \operatorname{HPFIGWBM}^{p, q}\left(\tilde{f}_{1}, \tilde{f}_{2}, \ldots, \tilde{f}_{n}\right) \\
& =\frac{1}{p+q} \otimes_{i, j=1, i \neq j}^{n}\left(p \tilde{f}_{i}^{w_{i}} \oplus q \tilde{f}_{j}^{w_{j}}\right)^{\frac{1}{n(n-1)}} \\
& =\bigcup_{\mu_{i k_{i}} \in \tilde{h}_{i}, v_{i k_{i}} \in \tilde{g}_{i}, \mu_{j k_{j}} \in \tilde{h}_{j}, v_{j k_{j}} \in \tilde{g}_{j}} \\
& \left\{\left(\left(1-\left(1-\prod_{i, j=1, i \neq j}^{n}\left(1-\left(1-\left(1-v_{i k_{i}}^{2}\right)^{w_{i}}\right.\right.\right.\right.\right.\right. \\
& \left.+\left(1-\left(\mu_{i k_{i}}^{2}+v_{i k_{i}}^{2}\right)\right)^{w_{i}}\right)^{p}\left(1-\left(1-v_{j k_{j}}^{2}\right)^{w_{j}}+\left(1-\left(\mu_{j k_{j}}^{2}\right.\right.\right. \\
& \left.\left.\left.+v_{j k_{j}}^{2}\right)\right)^{w_{j}}\right)^{q}+(1 \\
& \left.\left.-\left(\mu_{i k_{i}}^{2}+v_{i k_{i}}^{2}\right)\right)^{p w_{i}}\left(1-\left(\mu_{j k_{j}}^{2}+v_{j k_{j}}^{2}\right)\right)^{q w_{j}}\right)^{\frac{1}{n(n-1)}} \\
& +\prod_{i, j=1, i \neq j}^{n}\left(\left(1-\left(\mu_{i k_{i}}^{2}\right.\right.\right. \\
& \left.\left.\left.\left.\left.+v_{i k_{i}}^{2}\right)\right)^{p w_{i}}\left(1-\left(\mu_{j k_{j}}^{2}+v_{j k_{j}}^{2}\right)\right)^{q w_{j}}\right)^{\frac{1}{(n-1)}}\right)^{\frac{1}{p+q}}\right)^{1 / 2} \text {, } \\
& \left(\left(1-\prod_{i, j=1, i \neq j}^{n}(1-(1\right.\right. \\
& \left.-\left(1-v_{i k_{i}}^{2}\right)^{w_{i}}+\left(1-\left(\mu_{i k_{i}}^{2}+v_{i k_{i}}^{2}\right)\right)^{w_{i}}\right)^{p}(1 \\
& -\left(1-v_{j k_{j}}^{2}\right)^{w_{j}}+\left(1-\left(\mu_{j k_{j}}^{2}\right.\right. \\
& \left.\left.\left.+v_{j k_{j}}^{2}\right)\right)^{w_{j}}\right)^{q}+\left(1-\left(\mu_{i k_{i}}^{2}+v_{i k_{i}}^{2}\right)\right)^{p w_{i}}(1 \\
& \left.\left.-\left(\mu_{j k_{j}}^{2}+v_{j k_{j}}^{2}\right)\right)^{q w_{j}}\right)^{\frac{1}{n(n-1)}} \\
& +\prod_{i, j=1, i \neq j}^{n}\left(\left(1-\left(\mu_{i k_{i}}^{2}+v_{i k_{i}}^{2}\right)\right)^{p w_{i}}(1\right. \\
& \left.\left.\left.-\left(\mu_{j k_{j}}^{2}+v_{j k_{j}}^{2}\right)\right)^{q w_{j}}\right)^{\frac{1}{n(n-1)}}\right)^{\frac{1}{p+q}}- \\
& \prod_{i, j=1, i \neq j}^{n}\left(\left(1-\left(\mu_{i k_{i}}^{2}+v_{i k_{i}}^{2}\right)\right)^{p w_{i}}(1\right. \\
& \left.\left.\left.\left.\left.-\left(\mu_{j k_{j}}^{2}+v_{j k_{j}}^{2}\right)\right)^{q w_{j}}\right)^{\frac{1}{n(n-1)(p+q)}}\right)^{1 / 2}\right)\right\} .
\end{aligned}
$$

\section{An approach to Pythagorean fuzzy multiple attribute decision-making based on new interaction aggregation operators}

Suppose there is a multiple attribute decision-making problem. $\left\{A_{1}, A_{2}, \ldots, A_{m}\right\}$ is the alternative set, $\left\{C_{1}, C_{2}, \ldots, C_{n}\right\}$ is the attribute set. The experts evaluate alternatives with respect to attributes with Pythagorean fuzzy values. If they are familiar with the attributes, they can give evaluation values; if they are not familiar with attributes, they can refuse to give any evaluation values. Hence, the hesitant Pythagorean fuzzy decision matrix is formed. The proposed method based on the new hesitant Pythagorean fuzzy interaction aggregation operators is as follows.
Step 1. Decision-makers evaluate alternatives with respect to attributes with Pythagorean fuzzy values and hesitant Pythagorean fuzzy decision matrix is formed as $\tilde{D}=$ $\left(\tilde{f}_{i j}\right)_{m \times n}$.

Step 2. Calculate alternatives' collective evaluation values using the HPFIWBM operator or the HPFIGWBM operator using the following equations.

$$
\begin{aligned}
& \tilde{f}_{i}=\operatorname{HPFIWBM}^{p, q}\left(\tilde{f}_{i 1}, \tilde{f}_{i 2}, \ldots, \tilde{f}_{i n}\right) \\
& =\left(\frac{1}{n(n-1)} \oplus_{j, l=1, j \neq l}^{n}\left(\left(w_{j} \tilde{f}_{i j}\right)^{p} \otimes\left(w_{l} \tilde{f}_{i l}\right)^{q}\right)\right)^{\frac{1}{p+q}} \\
& = \\
& \mu_{i j k_{j}} \in \tilde{h}_{i j}, v_{i j k_{j}} \in \tilde{g}_{i j}, \mu_{i l k_{l}} \in \tilde{h}_{i l}, v_{i l k_{l}} \in \tilde{g}_{i l} \\
& \times\left\{\left(\left(\left(1-\prod_{j, l=1, j \neq l}^{n}\left(1-\left(1-\left(1-\mu_{i j k_{j}}^{2}\right)^{w_{j}}\right.\right.\right.\right.\right.\right. \\
& \left.+\left(1-\left(\mu_{i j k_{j}}^{2}+v_{i j k_{j}}^{2}\right)\right)^{w_{j}}\right)^{p}\left(1-\left(1-\mu_{i l k_{l}}^{2}\right)^{q}\right. \\
& \left.+\left(1-\left(\mu_{i l k_{l}}^{2}+v_{i l k_{l}}^{2}\right)\right)^{w_{l}}\right)^{q}+(1 \\
& \left.\left.-\left(\mu_{i j k_{j}}^{2}+v_{i j k_{j}}^{2}\right)\right)^{w_{j} p}\left(1-\left(\mu_{i l k_{l}}^{2}+v_{i l k_{l}}^{2}\right)\right)^{w_{l} q}\right)^{\frac{1}{n(n-1)}} \\
& +\prod_{j, l=1, j \neq l}^{n}\left(\left(1-\left(\mu_{i j k_{j}}^{2}\right.\right.\right. \\
& \left.\left.\left.\left.+v_{i j k_{j}}^{2}\right)\right)^{w_{j} p}\left(1-\left(\mu_{i l k_{l}}^{2}+v_{i l k_{l}}^{2}\right)\right)^{w_{l} q}\right)^{\frac{1}{(n-1)}}\right)^{\frac{1}{p+q}} \\
& -\prod_{j, l=1, j \neq l}^{n}\left(\left(1-\left(\mu_{i j k_{j}}^{2}\right.\right.\right. \\
& \left.\left.\left.\left.+v_{i j k_{j}}^{2}\right)\right)^{w_{j} p}\left(1-\left(\mu_{i l k_{l}}^{2}+v_{i l k_{l}}^{2}\right)\right)^{w_{l} q}\right)^{\frac{1}{n(n-1)(p+q)}}\right)^{1 / 2} \text {, } \\
& \times \quad\left(1-\left(1-\prod_{j, l=1, j \neq l}^{n}(1\right.\right. \\
& -\left(1-\left(1-\mu_{i j k_{j}}^{2}\right)^{w_{j}}+\left(1-\left(\mu_{i j k_{j}}^{2}+v_{i j k_{j}}^{2}\right)\right)^{w_{j}}\right)^{p} \\
& \times\left(1-\left(1-\mu_{i l k_{l}}^{2}\right)^{q}+(1\right. \\
& -\left(\mu_{i l k_{l}}^{2}\right. \\
& \left.\left.\left.+v_{i l k_{l}}^{2}\right)\right)^{w_{l}}\right)^{q}+\left(1-\left(\mu_{i j k_{j}}^{2}+v_{i j k_{j}}^{2}\right)\right)^{w_{j} p}(1 \\
& \left.\left.-\left(\mu_{i l k_{l}}^{2}+v_{i l j k_{l}}^{2}\right)\right)^{w_{l} q}\right)^{\frac{1}{(n-1)}} \\
& +\prod_{j, l=1, j \neq l}^{n}\left(\left(1-\left(\mu_{i j k_{j}}^{2}+v_{i j k_{j}}^{2}\right)\right)^{w_{j} p}(1\right. \\
& \left.\left.\left.\left.\left.\left.-\left(\mu_{i l k_{l}}^{2}+v_{i l k_{l}}^{2}\right)\right)^{w_{l} q}\right)^{\frac{1}{n(n-1)}}\right)^{\frac{1}{p+q}}\right)^{1 / 2}\right)\right\} \text {. }
\end{aligned}
$$$$
\tilde{f}_{i}=\operatorname{HPFIGWBM}^{p, q}\left(\tilde{f}_{i 1}, \tilde{f}_{i 2}, \ldots, \tilde{f}_{i n}\right)
$$$$
=\frac{1}{p+q} \otimes_{j, l=1, j \neq l}^{n}\left(p \tilde{f}_{i j}^{w_{j}} \oplus q \tilde{f}_{i l}^{w_{l}}\right)^{\frac{1}{n(n-1)}}
$$$$
=\quad \bigcup
$$$$
\mu_{i j k_{j}} \in \tilde{h}_{i j}, v_{i j k_{j}} \in \tilde{g}_{i j}, \mu_{i l k_{l}} \in \tilde{h}_{i l}, v_{i l k_{l}} \in \tilde{g}_{i l}
$$$$
\times\left\{\left(\left(1-\left(1-\prod_{j, l=1, j \neq l}^{n}\left(1-\left(1-\left(1-v_{i j k_{j}}^{2}\right)^{w_{j}}\right.\right.\right.\right.\right.\right.
$$$$
\left.+\left(1-\left(\mu_{i j k_{j}}^{2}+v_{i j k_{j}}^{2}\right)\right)^{w_{j}}\right)^{p}(1
$$ 
Table 1 Pythagorean fuzzy decision matrix $\tilde{D}$

\begin{tabular}{llll}
\hline & $C_{1}$ & $C_{2}$ & $C_{3}$ \\
\hline$A_{1}$ & $\{(0.9,0.2)\}$ & $\{(0.7,0.3)\}$ & $\{(0.6,0.5)\}$ \\
$A_{2}$ & $\{(0.5,0.6),(0.6,0.3)\}$ & $\{(0.8,0.3)\}$ & $\{(0.4,0.5)\}$ \\
$A_{3}$ & $\{(0.7,0)\}$ & $\{(0.6,0.2)\}$ & $\{(0.6,0.2),(0.8,0.2)\}$ \\
$A_{4}$ & $\{(0.7,0.4)\}$ & $\{(0.8,0.1),(0.7,0.4)\}$ & $\{(0.5,0.3)\}$ \\
\hline
\end{tabular}

$$
\begin{aligned}
& \left.-\left(1-v_{i l k_{l}}^{2}\right)^{w_{l}}+\left(1-\left(\mu_{i l k_{l}}^{2}+v_{i l k_{l}}^{2}\right)\right)^{w_{l}}\right)^{q}+(1 \\
& \left.-\left(\mu_{i j k_{j}}^{2}+v_{i j k_{j}}^{2}\right)\right)^{p w_{j}}\left(1-\left(\mu_{i l k_{l}}^{2}\right.\right. \\
& \left.\left.\left.+v_{i l k_{l}}^{2}\right)\right)^{q w_{l}}\right)^{\frac{1}{n(n-1)}}+\prod_{j, l=1, j \neq l}^{n}\left(\left(1-\left(\mu_{i j k_{j}}^{2}\right.\right.\right. \\
& \left.\left.\left.\left.\left.+v_{i j k_{j}}^{2}\right)\right)^{p w_{j}}\left(1-\left(\mu_{i l k_{l}}^{2}+v_{i l k_{l}}^{2}\right)\right)^{q w_{l}}\right)^{\frac{1}{n(n-1)}}\right)^{\frac{1}{p+q}}\right)^{1 / 2}, \\
& \times\left(\left(1-\prod_{j, l=1, j \neq l}^{n}(1-(1\right.\right. \\
& \left.-\left(1-v_{i j k_{j}}^{2}\right)^{w_{j}}+\left(1-\left(\mu_{i j k_{j}}^{2}+v_{i j k_{j}}^{2}\right)\right)^{w_{j}}\right)^{p}(1 \\
& -\left(1-v_{i l k_{l}}^{2}\right)^{w_{l}}+\left(1-\left(\mu_{i l k_{l}}^{2}\right.\right. \\
& \left.\left.\left.+v_{i l k_{l}}^{2}\right)\right)^{w_{l}}\right)^{q}+\left(1-\left(\mu_{i j k_{j}}^{2}+v_{i j k_{j}}^{2}\right)\right)^{p w_{j}}(1 \\
& \left.\left.-\left(\mu_{i l k_{l}}^{2}+v_{i l k_{l}}^{2}\right)\right)^{q w_{l}}\right)^{\frac{1}{(n-1)}} \\
& +\prod_{j, l=1, j \neq l}^{n}\left(( 1 - ( \mu _ { i j k _ { j } } ^ { 2 } + v _ { i j k _ { j } } ^ { 2 } ) ) ^ { p w _ { j } } \left(1-\left(\mu_{i l k_{l}}^{2}\right.\right.\right. \\
& \left.\left.\left.\left.+v_{i l k_{l}}^{2}\right)\right)^{q w_{l}}\right)^{\frac{1}{(n-1)}}\right)^{\frac{1}{p+q}} \\
& -\prod_{j, l=1, j \neq l}^{n}\left(( 1 - ( \mu _ { i j k _ { j } } ^ { 2 } + v _ { i j k _ { j } } ^ { 2 } ) ) ^ { p w _ { j } } \left(1-\left(\mu_{i l k_{l}}^{2}\right.\right.\right. \\
& \left.\left.\left.\left.\left.\left.+v_{i l k_{l}}^{2}\right)\right)^{q w_{l}}\right)^{\frac{1}{n(n-1)(p+q)}}\right)^{1 / 2}\right)\right\} \text {, }
\end{aligned}
$$

where $\left(w_{1}, w_{2}, \ldots, w_{n}\right)$ is the weight vector of different attributes with $w_{i} \geq 0$ and $\sum_{i=1}^{n} w_{i}=1$.

Step 3. Calculate each alternative's $S\left(\tilde{f}_{i}\right)$ and $A\left(\tilde{f}_{i}\right)$ using the Eqs. (4)-(5).

Step 4. Rank alternatives according to the method in Definition 7.

The new method has the following characteristics: the evaluation values are given as HPFEs, which are more flexible and powerful; interaction between membership and nonmembership has been considered; and interaction between arguments to be aggregated has been modeled using the Bonferroni mean operator.

\section{An illustrative example}

Suppose there is an investing company wanting to invest a large amount of money (adapted from [57]). They invite several experts to evaluate several possible companies: $A_{1}$-an artificial intelligent company, $A_{2}$ - an architecture company, $A_{3}$ - a catering company and $A_{4}$-a logistics company. They mainly consider the following attributes: $C_{1}$-interest rate, $C_{2}$-risk, $C_{3}$ - growth potential. The new method is used to rank alternatives as follows.

Step 1. The experts evaluate alternatives with respect to attributes in Pythagorean fuzzy values and the decision matrix is formed as $\tilde{D}=\left(\tilde{f}_{i j}\right)_{4 \times 3}$ (Table 1).

Step 2. Assume the weight vector of attributes is $(0.45,0.35$, $0.20)$. Calculate the collective evaluation values by using the HPFIWBM $^{2,2}$ operator to get

$$
\tilde{f}_{1}=\{(0.5605,0.2174)\}, \tilde{f}_{2}=\{(0.4238,0.3176),
$$
$(0.4240,0.2861),(0.4368, \quad 0.2272),(0.4392,0.2623)\}$, $\tilde{f}_{3}=\{(0.4477,0.1012),(0.4995,0.0880),(0.4975,0.0988)$, $(0.5420,0.0862)\}, \quad \tilde{f}_{4}=\{(0.4486,0.2522),(0.4621$, $0.2264),(0.4638,0.2229),(0.4753,0.1972)\}$.

Step 3. The scores of $\tilde{f}_{i}$ are calculated as

$S\left(\tilde{f}_{1}\right)=0.2670, \quad S\left(\tilde{f}_{2}\right)=0.1100$,

$S\left(\tilde{f}_{3}\right)=0.2515, \quad S\left(\tilde{f}_{4}\right)=0.1631$.

Step 4. The alternatives can be ranked as

$A_{3}>A_{1}>A_{4}>A_{2}$.

The optimal alternative is $A_{3}$.

\section{Comparing with other methods}

If the hesitant Pythagorean fuzzy interaction weighted averaging (HPFIWA) operator is used in aggregating, we can get $\tilde{f}_{1}=\{(0.8449,0.3123)\}, \tilde{f}_{2}=\{(0.6979,0.4759)$, $(0.7228,0.3785)\}, \tilde{f_{3}}=\{(0.7864,0.1734),(0.7111$, $0.1670)\}, \tilde{f}_{4}=\{(0.7683,0.2955),(0.7216,0.1670)\}$. The scores of $\tilde{f}_{i}(i=1,2, \ldots, 4)$ can be calculated as $S\left(\tilde{f}_{1}\right)=$ 0.6163, $S\left(\tilde{f}_{2}\right)=0.3199, S\left(\tilde{f}_{3}\right)=0.5331, S\left(\tilde{f}_{4}\right)=0.4334$. Then alternatives can be ranked as $A_{1}>A_{3}>A_{4}>A_{2}$ and the optimal alternative is $A_{1}$. The ranking result is different from that based on the HPFIWBM operator. Though the interaction between membership and non-membership has been considered, interaction between hesitant Pythagorean fuzzy elements has not been considered. 


$$
\begin{aligned}
& \operatorname{HPFIWA}\left(\tilde{f}_{1}, \tilde{f}_{2}, \ldots, \tilde{f}_{n}\right) \\
& =\bigcup_{\mu_{i k_{i}} \in \tilde{h}_{i}, v_{i k_{i}} \in \tilde{g}_{i}} \\
& \quad \times\left\{\left(\sqrt{1-\prod_{i=1}^{n}\left(1-\mu_{i k_{i}}^{2}\right)^{w_{i}}},\left(\prod_{i=1}^{n}\left(1-\mu_{i k_{i}}^{2}\right)^{w_{i}}\right.\right.\right. \\
& \left.\left.\left.\quad-\prod_{i=1}^{n}\left(1-\left(\mu_{i k_{i}}^{2}+v_{i k_{i}}^{2}\right)\right)^{w_{i}}\right)^{1 / 2}\right)\right\} .
\end{aligned}
$$

If interaction between membership and non-membership is not considered and we calculate the collective ones using the hesitant Pythagorean fuzzy weighted averaging (HPFWA) operator as follows

$$
\begin{aligned}
\operatorname{HPFWA} & \left(\tilde{f}_{1}, \tilde{f}_{2}, \ldots, \tilde{f}_{n}\right) \\
= & \bigcup_{\mu_{i k_{i}} \in \tilde{h}_{i}, v_{i k_{i}} \in \tilde{g}_{i}} \\
& \times\left\{\left(\sqrt{\left.\left.1-\prod_{i=1}^{n}\left(1-\mu_{i k_{i}}^{2}\right)^{w_{i}}, \prod_{i=1}^{n} v_{i k_{i}}^{w_{i}}\right)\right\} .}\right.\right.
\end{aligned}
$$

We can calculate collective evaluation values using the HPFWA operator to get $\tilde{f}_{1}=\{(0.8110,0.2769)\}, \tilde{f}_{2}=$ $\{(0.6377,0.4539),(0.6689,0.3323)\}, \tilde{f}_{3}=\{(0.6964,0)$, $(0.6951,0)\}, \tilde{f}_{4}=\{(0.6964,0.3446),(0.7158,0.2325)\}$. The scores can be calculated as $S\left(\tilde{f}_{1}\right)=0.5811, S\left(\tilde{f}_{2}\right)=$ $0.2688, S\left(\tilde{f}_{3}\right)=0.4841, S\left(\tilde{f}_{4}\right)=0.4123$. Then, alternatives can be ranked as $A_{1}>A_{3}>A_{4}>A_{2}$ and the optimal alternative is $A_{1}$. From the result, we can see that the aggregated non-membership of $A_{3}$ is 0 due to the 0 nonmembership in evaluation values. Though all the other non-memberships are not 0 , they have no effect on the final result.

If the TOPSIS method is used to rank alternatives, we first extend decision matrix by adding the minimum Pythagorean fuzzy value $(0.4,0.5)$ to make all the evaluation values have the same number of membership and non-membership. The hesitant Pythagorean fuzzy positive ideal solution $\tilde{f}^{+}$and hesitant Pythagorean fuzzy negative ideal solution $\tilde{f}^{-}$can be determined as $\tilde{f}^{+}=(\{(0.9,0.2),(0.4,0.5)\},\{(0.8,0.1)$, $(0.7,0.4)\},\{(0.8,0.1),(0.7,0.4)\},\{(0.8,0.2),(0.6,0.2)\})$, $\tilde{f}^{-}=(\{(0.5,0.6),(0.6,0.3)\},\{(0.6,0.2),(0.4,0.5)\},\{(0.6$, $0.2),(0.4,0.5)\},\{(0.4,0.5),(0.4,0.5)\})$. The weighted distances between alternative evaluation values and $\tilde{f}^{+}, \tilde{f}^{-}$ can be calculated using the following equations $d_{i}^{+}=$ $w_{1} d\left(\tilde{f}_{1}^{+}, \tilde{f}_{i 1}\right)+w_{2} d\left(\tilde{f}_{2}^{+}, \tilde{f}_{i 2}\right)+w_{3} d\left(\tilde{f}_{3}^{+}, \tilde{f}_{i 3}\right), d_{i}^{-}=$ $w_{1} d\left(\tilde{f}_{1}^{-}, \tilde{f}_{i 1}\right)+w_{2} d\left(\tilde{f}_{2}^{-}, \tilde{f}_{i 2}\right)+w_{3} d\left(\tilde{f}_{3}^{-}, \tilde{f}_{i 3}\right)$. The results are as $d_{1}^{+}=0.0301, d_{2}^{+}=0.0495, d_{3}^{+}=0.0834$, $d_{4}^{+}=0.0390, d_{1}^{-}=0.1653, d_{2}^{-}=0.0289, d_{3}^{-}=0.1630$, $d_{4}^{-}=0.1663$. The closeness coefficients can be calculated by $C C_{i}=\frac{d_{i}^{-}}{d_{i}^{-}+d_{i}^{+}}$to get $C C_{1}=0.8458, C C_{2}=$ $0.3684, C C_{3}=0.6616, C C_{4}=0.8101$. Then alternatives can be ranked as $A_{1}>A_{4}>A_{3}>A_{2}$ and the optimal alternative is $A_{1}$.

If interaction between memberships and non-memberships is not considered, the hesitant Pythagorean fuzzy weighted Bonferroni mean (HPFWBM) operator as follows is used in aggregating.

$$
\begin{aligned}
& \operatorname{HPFWBM}\left(\tilde{f}_{1}, \tilde{f}_{2}, \ldots, \tilde{f}_{n}\right) \\
& =\left(\frac{1}{n(n-1)} \oplus_{i, j=1, i \neq j}^{n}\left(\left(w_{i} \tilde{f}_{i}\right)^{p} \otimes\left(w_{j} \tilde{f}_{j}\right)^{q}\right)\right)^{\frac{1}{p+q}} \\
& =\bigcup_{\mu_{j k_{j}} \in \tilde{h}_{j}, v_{j k_{j}} \in \tilde{g}_{j}} \\
& \quad \times\left\{\left(\sqrt{\left(1-\prod_{i, j=1, i \neq j}^{n}\left(1-\left(1-\left(1-\mu_{j k_{j}}^{2}\right)^{w_{j}}\right)^{q}\right)^{\frac{1}{n(n-1)}}\right)^{\frac{1}{p+q}}},\right.\right. \\
& \left.\left.\quad \times \sqrt{\left(1-\left(1-\prod_{i, j=1, i \neq j}^{n}\left(1-\left(1-\left(v_{j k_{j}}^{w_{j}}\right)^{2}\right)^{q}\right)^{\frac{1}{n(n-1)}}\right)^{\frac{1}{p+q}}\right.}\right)\right\} .
\end{aligned}
$$

The collective evaluation values can be calculated as $\tilde{f}_{1}=$ $\{(0.4713,0.7377)\}, \tilde{f}_{2}=\{(0.3533,0.8181),(0.3886$, $0.7650),(0.3540,0.8065), \quad(0.3889,0.7585)\}, \quad \tilde{f}_{3}=$ $\{(0.4066,0.6147),(0.3806,0.6147),(0.3998,0.6147)$, $(0.3694,0.6147)\}, \tilde{f}_{4}=\{(0.4275,0.7107),(0.3944,0.7517)$, $(0.4263,0.7264),(0.3923,0.7753)\}$. The scores can be calculated as $S\left(\tilde{f}_{1}\right)=-0.3221, S\left(\tilde{f}_{2}\right)=-0.4820, S\left(\tilde{f}_{3}\right)=$ $-0.2257, S\left(\tilde{f}_{4}\right)=-0.7377$. The alternatives can be ranked as $A_{3}>A_{1}>A_{4}>A_{2}$ and the optimal alternative is $A_{3}$. Though ranking result is the same as that based on the HPFIWBM operator, but in aggregation process, the effect of non-memberships has been reduced since there is 0 of non-membership in the evaluation process. In other decisionmaking problems, we may get different ranking results.

The differences between the proposed method and the existing methods have been summarized in Table 2. In a word, our proposed method is based on the hesitant Pythagorean fuzzy values and the Bonferroni mean operator. Moreover, interaction between arguments to be aggregated is considered and interaction between membership and non-membership is also considered.

\section{Conclusions}

In this paper, we first define some hesitant Pythagorean fuzzy interaction aggregation laws for HPFEs, and then develop some hesitant Pythagorean interaction aggregation operators. Using the Bonferroni mean operator, we develop 
Table 2 The characteristic comparisons of different methods

\begin{tabular}{lll}
\hline Methods & $\begin{array}{l}\text { Information by Pythagorean } \\
\text { fuzzy number }\end{array}$ & $\begin{array}{l}\text { Whether consider the inter- } \\
\text { relationships between aggre- } \\
\text { gating arguments }\end{array}$ \\
\hline Liang et al. [22] & Yes & Yes \\
Xu and Yager [58] & No & Yes \\
Zhang and Xu [32] & Yes & No \\
Garg [50] & Yes & No \\
Our proposed method & Yes & Yes \\
\hline Methods & Whether consider the inter- & Whether information by \\
& actions between member- & hesitant Pythagorean fuzzy \\
& ship and non-membership \\
\hline Liang et al. [22] & No & No \\
Xu and Yager [58] & No & No \\
Zhang and Xu [32] & No & No \\
Garg [50] & Yes & Yes \\
Our proposed method & Yes & Yes \\
\hline
\end{tabular}

some hesitant Pythagorean fuzzy interaction Bonferroni mean operators. Based on the HPFIWBM operator and the HPFIWGBM operator, we propose a new multiple attribute decision-making method. Numerical example is presented to illustrate the new method.

In the future, we will apply the new aggregation operators to other complicated decision problems and we will also develop new interaction aggregation operators for HPFEs.

Funding This work is partly supported by National Natural Science Foundation of China (no. 11401457), Postdoctoral Science Foundation of China (no. 2015M582624), Shaanxi Province Postdoctoral Science Foundation of China and Department of Education Fund of Shaanxi Province, China (no. 17JK0440).

\section{Compliance with ethical standards}

Conflict of interest The authors declare that there is no conflict of interests regarding the publication of this paper.

Open Access This article is distributed under the terms of the Creative Commons Attribution 4.0 International License (http://creativecomm ons.org/licenses/by/4.0/), which permits unrestricted use, distribution, and reproduction in any medium, provided you give appropriate credit to the original author(s) and the source, provide a link to the Creative Commons license, and indicate if changes were made.

\section{References}

1. Merigó JM, Gil-Lafuente AM, Yager RR (2015) An overview of fuzzy research with bibliometric indicators. Appl Soft Comput 27:420-433

2. Blanco-Mesa F, Merigó JM, Gil-Lafuente AM (2017) Fuzzy decision making: a bibliometric-based review. J Intell Fuzzy Syst 32(3):2033-2050
3. Merigó JM (2010) Fuzzy decision making with immediate probabilities. Comput Ind Eng 58(4):651-657

4. Yager RR (2013) Pythagorean fuzzy subsets. In: Proceedings of the joint IFSA World congress and NAFIPS annual meeting, Edmonton, Canada, pp 57-61

5. Yager RR, Abbasov AM (2013) Pythagorean membership grades, complex numbers, and decision making. In J Intell Syst 28:436452

6. Atanassov KT (1986) Intuitionistic fuzzy sets. Fuzzy Sets Syst 20:87-96

7. Wei GW, Garg H, Gao H, Wei C (2018) Interval-valued Pythagorean fuzzy Maclaurin symmetric mean operators in multiple attribute decision making. IEEE Access. https://doi.org/10. 1109/ACCESS.2018.2877725

8. Garg H (2019) New Logarithmic operational laws and their aggregation operators for Pythagorean fuzzy set and their applications. Int J Intell Syst 34(1):82-106

9. Garg H (2018) New exponential operational laws and their aggregation operators for interval-valued Pythagorean fuzzy multicriteria decision-making. Int J Intell Syst 33(3):653-683

10. Garg H (2018) A linear programming method based on an improved score function for interval-valued Pythagorean fuzzy numbers and its application to decision-making. Int J Uncertain Fuzziness Knowl Based Syst 29(1):67-80

11. Garg H (2017) Confidence levels based Pythagorean fuzzy aggregation operators and its application to decision-making process. Comput Math Org Theory 23(4):546-571

12. Garg H (2017) A new improved score function of an interval-valued Pythagorean fuzzy set based TOPSIS method. Int J Uncertain Quantif 7(5):463-474

13. Garg H (2017) A novel improved accuracy function for intervalvalued Pythagorean fuzzy sets and its applications in decision making process. Int J Intell Syst 32(12):1247-1260

14. Garg H (2018) Hesitant Pythagorean fuzzy Maclaurin symmetric mean operators and its applications to multiattribute decision making process. Int J Intell Syst. https://doi.org/10.1002/int.22067

15. Yang W, Pang YF (2019) Hesitant Interval-valued Pythagorean Fuzzy VIKOR Method. International J Intell Syst 34(5):754-789

16. Yager RR (2014) Pythagorean membership grades in multicriteria decision making. IEEE Trans Fuzzy Syst 22(4):958-965

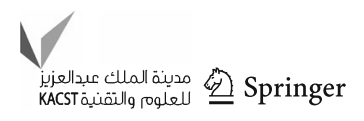


17. Yager RR (2016) Properties and applications of Pythagorean fuzzy sets. Stud Fuzziness Soft Comput 332:119-136

18. Dick S, Yager YY, Yazdanbakhsh O (2016) On Pythagorean and complex fuzzy set operations. IEEE Trans Fuzzy Syst 24(5):10091021

19. Reformat MZ, Yager RR (2017) Composition-based Users' matching processes with Pythagorean fuzzy sets. In: 2017 IEEE international conference on fuzzy systems, 1-6 July

20. Peng XD, Selvachandran G (2017) Pythagorean fuzzy set: state of the art and future directions. Artif Intell Rev. https://doi.org/10. 1007/s10462-017-9596-9

21. Peng XD, Yang Y (2015) Some results for Pythagorean fuzzy sets. Int J Intell Syst 30:1133-1160

22. Liang DC, Zhang YRJ, Xu ZS, Darko AP (2018) Pythagorean fuzzy Bonferroni mean aggregation operator and its accelerative calculating algorithm with the multithreading. Int J Intell Syst 33(3):615-633

23. Zhang RT, Wang J, Zhu XM, Xia MM, Yu M (2017) Some generalized Pythagorean fuzzy Bonferroni mean aggregation operators with their application to multiattribute group decision-making. Complexity. https://doi.org/10.1155/2017/5937376

24. Yang W, Pang YF (2018) New Pythagorean fuzzy interaction Maclaurin symmetric mean operators and their application in multiple attribute decision making. IEEE Access. https://doi.org/10. 1109/ACCESS.2018.2856270

25. Rahman K, Abdullah S, Shakeel M, Khan MSA, Ullah M (2017) Interval-valued Pythagorean fuzzy geometric aggregation operators and their application to group decision making problem. Cogent Math 4(1): 1338638

26. Wei GW, Lu M (2018) Pythagorean fuzzy power aggregation operators in multiple attribute decision making. Int J Intell Syst 33:169-186

27. Wei GW, Lu M (2017) Dual hesitant Pythagorean fuzzy Hamacher aggregation operators in multiple attribute decision making. Archi Control Sci 27(3):365-395

28. Garg H (2016) A new generalized Pythagorean fuzzy information aggregation using Einstein operations and its application to decision making. Int J Intell Syst 31:886-920

29. Garg H (2018) Generalised Pythagorean fuzzy geometric interactive aggregation operators using Einstein operations and their application to decision making. J Exp Theor Artif Intell 30(6):763794

30. Du YQ, Hou F, Zafar W, Yu Q, Zhai Y (2017) A novel method for multiattribute decision making with interval-valued Pythagorean fuzzy linguistic information. Int J Intell Syst 32(10):1085-1112

31. Wei GW (2017) Pythagorean fuzzy interaction aggregation operators and their application to multiple attribute decision making. J Intell Fuzzy Syst 33(4):2119-2132

32. Zhang XL, Xu ZS (2014) Extension of TOPSIS to multiple criteria decision making with Pythagorean fuzzy set. Int J Intell Syst 29:1061-1078

33. Ren PJ, Xu ZS, Gou XJ (2016) Pythagorean fuzzy TODIM approach to multi-criteria decision making. Appl Soft Comput 42:246-259

34. Chen TY (2018) Remoteness index-based Pythagorean fuzzy VIKOR methods with a generalized distance measure for multiple criteria decision analysis. Inf Fusion 41:129-150

35. Liang W, Zhang XL, Liu MF (2015) The maximizing deviation method based on interval-valued Pythagorean fuzzy weighted aggregating operator for multiple criteria group decision analysis. Discrete Dyn Nat Soc. https://doi.org/10.1155/2015/746572

36. Garg H (2016) A novel accuracy function under interval-valued Pythagorean fuzzy environment for solving multicriteria decision making problem. J Intell Fuzzy Syst 31:529-540

37. Liu Z, Liu P, Liu W, Pang J (2017) Pythagorean uncertain linguistic partitioned Bonferroni mean operators and their application in multi-attribute decision making. J Intell Fuzzy Syst 32(3):2779_ 2790

38. Peng XD, Yang Y (2016) Multiple attribute group decision making methods based on Pythagorean fuzzy linguistic set. Comput Eng 52(23):50-54

39. Zeng SZ (2017) Pythagorean fuzzy multiattribute group decision making with probabilistic information and OWA approach. Int $\mathbf{J}$ Intell Syst 32:1136-1150

40. Torra V (2010) Hesitant fuzzy sets. Int J Intelli Syst 25:529-539

41. Peng JJ, Wang JQ, Wu XH, Zhang HY, Chen XH (2015) The fuzzy cross-entropy for intuitionistic hesitant fuzzy sets and their application in multi-criteria decision-making. Int J Syst Sci 46(13):2335-2350

42. Rodríguez RM, Martínez L, Herrera F (2012) Hesitant fuzzy linguistic term sets for decision making. IEEE Trans Fuzzy Syst 20(1):109-119

43. Yang W, Pang YF, Shi JR, Wang CJ (2018) Linguistic hesitant intuitionistic fuzzy decision-making method based on VIKOR. Neural Comput Appl 29:613-626

44. Yang W, Pang YF, Shi JR, Yue HY (2017) Linguistic hesitant intuitionistic fuzzy linear assignment method based on Choquet integral. J Intell Fuzzy Syst 32:767-780

45. Yang W, Pang YF, Shi JR (2017) Linguistic hesitant intuitionistic fuzzy cross-entropy measures. Int J Comput Intell Syst 10:120-139

46. Yang W, Shi JR, Zheng XY, Pang YF (2016) Hesitant intervalvalued intuitionistic fuzzy linguistic sets and their applications. J Intell Fuzzy Syst 31:2779-2788

47. Yang W, Shi JR, Pang YF (2015) Generalized linguistic hesitant intuitionistic fuzzy hybrid aggregation operators. Math Probl Eng 1-11:2015. https://doi.org/10.1155/2015/983628

48. Lu M, Wei GW, Alsaadi FE, Hayat T, Alsaedi A (2017) Hesitant Pythagorean fuzzy Hamacher aggregation operators and their application to multiple attribute decision making. J Intell Fuzzy Syst 33(2):1105-1117

49. Khan MSA, Abdullah S, Ali A (2017) Pythagorean hesitant fuzzy sets and their application to group decision making with incomplete weight information. J Intell Fuzzy Syst 33(6):3971-3985

50. Garg H (2018) Hesitant Pythagorean fuzzy sets and their aggregation operators in multiple attribute decision-making. Int J Uncertain Quantif 8(3):267-289

51. Bonferroni C (1950) Sulle medie multiple dipotenze. Boll Mat Ital 5:267-270

52. Yager RR (2009) On generalized Bonferroni mean operators for multi-criteria aggregation. Int J Approx Reason 50:1279-1286

53. Beliakov G, James S, Mordelova J, Ruckschlossova T, Yager RR (2010) Generalized Bonferroni mean operators in multi-criteria aggregation. Fuzzy Sets Syst 161(17):2227-2242

54. Beliakov G, James S (2013) On extending generalized Bonferroni means to Atanassov orthopairs in decision making contexts. Fuzzy Sets Syst 211:84-98

55. Zhu B, Xu ZS (2013) Hesitant fuzzy Bonferroni means for multicriteria decision making. J Oper Res Soc 64:1831-1840

56. Yang W, Shi JR, Liu Y, Pang YF, Lin RY (2018) Pythagorean fuzzy interaction partitioned Bonferroni mean operators and their application in multiple attribute decision making. Complexity 2018:1-25. https://doi.org/10.1155/2018/3606245

57. Yang W, Pang YF (2019) New q-rung orthopair fuzzy partitioned Bonferroni mean operators and their application in multiple attribute decision making. Int J Intell Syst 34(3):439-476

58. Xu ZS, Yager RR (2011) Intuitionistic fuzzy Bonferroni means. IEEE Trans Syst Man Cybern Part B Cybern 41(2):568-578

Publisher's Note Springer Nature remains neutral with regard to jurisdictional claims in published maps and institutional affiliations.
1

مدينة الملك عبدالعزيز

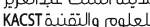

